\title{
Stilt bugs (Hemiptera: Heteroptera: Berytidae) of Poland: check-list, distribution, bionomics
}

\author{
Grzegorz GIERLASIŃSKI ${ }^{1}$, Artur TASZAKOWSKI ${ }^{2}$ and Barbara LIS ${ }^{3}$ \\ ${ }^{I}$ Doliny Miętusiej 27/44, 43-316 Bielsko-Biała, Poland; e-mail: ggierlas@gmail.com (corresponding author) \\ ${ }^{2}$ Department of Zoology, Faculty of Biology and Environmental Protection, University of Silesia in Katowice, \\ Bankowa 9, 40-007 Katowice, Poland; e-mail: artur.taszakowski@us.edu.pl \\ ${ }^{3}$ Department of Biosystematics, University of Opole, ul. Oleska 22, 45-052 Opole, Poland; e-mail: canta@uni.opole.pl
}

\begin{abstract}
In Poland, the family Berytidae Fieber, 1851 is currently represented by nine species. This paper gives an overview of their biology, feeding habits, hosts, and known distribution, and presents new localities and the review of their distribution in Poland.
\end{abstract}

Key words: true bugs, faunistics, biodiversity, ecology

\section{INTRODUCTION}

The family Berytidae belongs to the infraorder Pentatomomorpha, superfamily Lygaeoidea, and includes three subfamilies, i.e. Berytinae, Gampsocorinae and Metacanthinae (Péricart 2001).

Representatives of the family Berytidae are small or medium-sized insects, reaching from 2.30 to over $16 \mathrm{~mm}$ in length. They are characterized by a narrow, highly elongated, rodshaped body (except for some taxa occurring in the Americas) and highly elongated legs and antennae. Body colouration is yellowish, yellowish-brown, brown, gray-brown or, rarely, redbrown. Until recently, species representing this family have been classified as typical phytophagous species, but recent studies (Henry 2000, Morkel 2007) have shown that these insects supplement their diet with animal food - they can actively hunt small insects (for example, aphids) or suck dead ones, or scavenge spider webs. Representatives of the genus Berytinus Kirkaldy, 1900 feed mainly on plants of the family Fabaceae, whereas representatives of the other genera prefer plants covered with glandular hairs (Cucurbitaceae, Geraniaceae, Lamiaceae, Malvaceae, Scrophulariaceae, Solanaceae). Most probably, they seek and suck up small prey trapped in the viscous secretions produced by these plants. All species occuring in Poland overwinter as adults.

In Poland, Berytidae are of no economic importance, because there are few species, which only sporadically occur on crop plants. That is why they are rarely collected by entomologists. Hence, data on the occurrence of Berytidae in Poland are scattered across many papers, which sometimes are very old and difficult to access. This prompted us to collect, review and summarize all these data in the present paper.

\section{MATERIAL AND METHODS}

We based the preparation of the manuscript on literature data, material deposited in four Polish institutional collections (i.e. Department of Zoology, Faculty of Biology and Environmental Protection, University of Silesia in Katowice (DZUS); Upper Silesian Museum in Bytom (USMB); Department of Biosystematics, University of Opole (DBUO); Museum and 
Institute of Zoology PAS, Warsaw (ZMPA)), information coming from the entomological forum (http://www.entomo.pl) and our own observations.

The borders of the geographical regions of Poland follow the Catalogue of the Polish Fauna (Burakowski et al. 1973).

Because most commonly identified chorological elements do not adequately describe the diversity of Heteroptera geographic ranges (Taszakowski \& Gorczyca 2018), for the purpose of the present paper, we used the zoogeographical elements proposed by Mazur (2001). Detailed principles for defining the chorological elements of Heteroptera were provided by Taszakowski \& Gorczyca (2018).

Data on the bionomics of the presented species were based on papers by Péricart (1984), Henry \& Froeschner (1998), B. Lis (2007) and Wachmann et al. (2007).

Distribution maps of the Berytidae species in Poland were generated using non-commercial software MapaUTM v5.2 (http://www.heteroptera.us.edu.pl/mapautm.html). Colour images were captured with a Leica DFC495 camera mounted on a Leica M205C stereomicroscope using a Leica application suite 4.9.0 software, and an Adobe Photoshop CS6 graphic editor.

Abbreviations used in the text: mt. - mountain, mts - mountains, massif, n. - near; LP landscape park; NP - national park; res. - reserve; val. - valley; asterisk (*) marks unpublished data.

\section{SYSTEMATIC CHECK-LIST OF THE STILT BUGS OF POLAND}

Berytinae Fieber, 1851

Berytini Fieber, 1851

\section{Neides tipularius (Linnaeus, 1758)}

Neides favosus: Smreczyński 1906a:74, 1954: 78.

Neides tipularius f. immaculata: Smreczyński 1954: 78.

Berytus tipularius: Siebold 1839: 433; Scholtz 1847: 151; Ziarkiewicz 1962: 100.

Range element: Caspian-Atlantic.

Bionomics: Mesophilous/xerothermophilous; polyphagous (mainly Caryophyllaceae, Geraniaceae, Asteraceae, Scrophulariaceae and Poaceae); one generation a year.

Remarks: Common and widely distributed in Poland.

Distribution in Poland (Fig. 1):

Baltic Coast: Chłapowo [CF37] - Smreczyński 1954; Gdynia [CF44] - Lis B. \& Kowalczyk 2017; Karpinka [VV88] - Schmidt 1928; *Smołdzino [XA46] - 1 Aug 2002, 1 ex., leg. B. \& J. A. Lis (DBUO);

Białowieża Forest: Czerlonka [FD84] - Strawiński 1956b; general [FD94] - Trojan et al. 1994.

Eastern Beskidy Mts: Bednarka [EA20, EV29] - Taszakowski \& Gorczyca 2018; *Bednarka, Cieklinka Mt. [EV29] - 6 Aug 2015, 1 ex., leg. R. Dobosz (USMB); Ciężkowice [DA91] - Smreczyński 1954; Libusza [EA10] - Taszakowski \& Gorczyca 2018; Przemyśl, Lipowica [FA21] - Kotula 1890; Przemyśl, Wzniesienie [FA21] - Kotula 1890; Zarzecze [FA13] - Krasucki 1919.

Eastern Sudetes Mts: *Gipsowa Góra Res. [YR14] - 8 Sep 1932, 4 exx., leg. H. Nowotny (USMB).

Krakowsko-Wieluńska Upland: Błędowska Desert [CA97] - Lis J. A. 1989, Lis J. A. \& Lis B. 1998; Czatkowice [DA05] - Smreczyński 1906b; Czyżówki [DA16] - Chłond \& 
Gorczyca 2009; Kraków [DA24] - Smreczyński 1954; Kraków, Bielany [DA14] - Stobiecki 1886, Smreczyński 1906b; Kraków, Błonia [DA24] - Stobiecki 1886; Kraków, Borek Fałęcki [DA24] - Smreczyński 1906b, Stobiecki 1915; Kraków, Dębniki [DA14] - Stobiecki 1886; Kraków, Krzemionki [DA14] - Stobiecki 1886, Smreczyński 1906b, Stobiecki 1915; Kraków, Mydlniki [DA24] - Stobiecki 1915; Kraków, Olsza [DA24] - Smreczyński 1906b; Kraków, Panieńskie Skały [DA14] - Stobiecki 1886; Kraków, Podgórki [DA14] - Smreczyński 1954; Kraków, Podgórze [DA24] - Stobiecki 1886; Kraków, Przegorzały [DA14] - Stobiecki 1915; Kraków, Sikornik [DA14] - Stobiecki 1886; *Mirów [DB00] - 21 Jul 1999, 1 ex., leg. A. Pańczyk (DBUO); *Podlesice, n. Zborów Mt. [CB90] - 11 Jul 2006, 5 exx., leg. D. Kolbe (DZUS); Przedmość [CB16, CB26] - Noga \& Lis B. 2015; Rudawa [DA05] - Stobiecki 1915; Rząska [DA15] - Smreczyński 1906b; Wierzchowie [DA15] - Smreczyński 1954; Zabierzów [DA15] - Stobiecki 1886, Smreczyński 1906b.

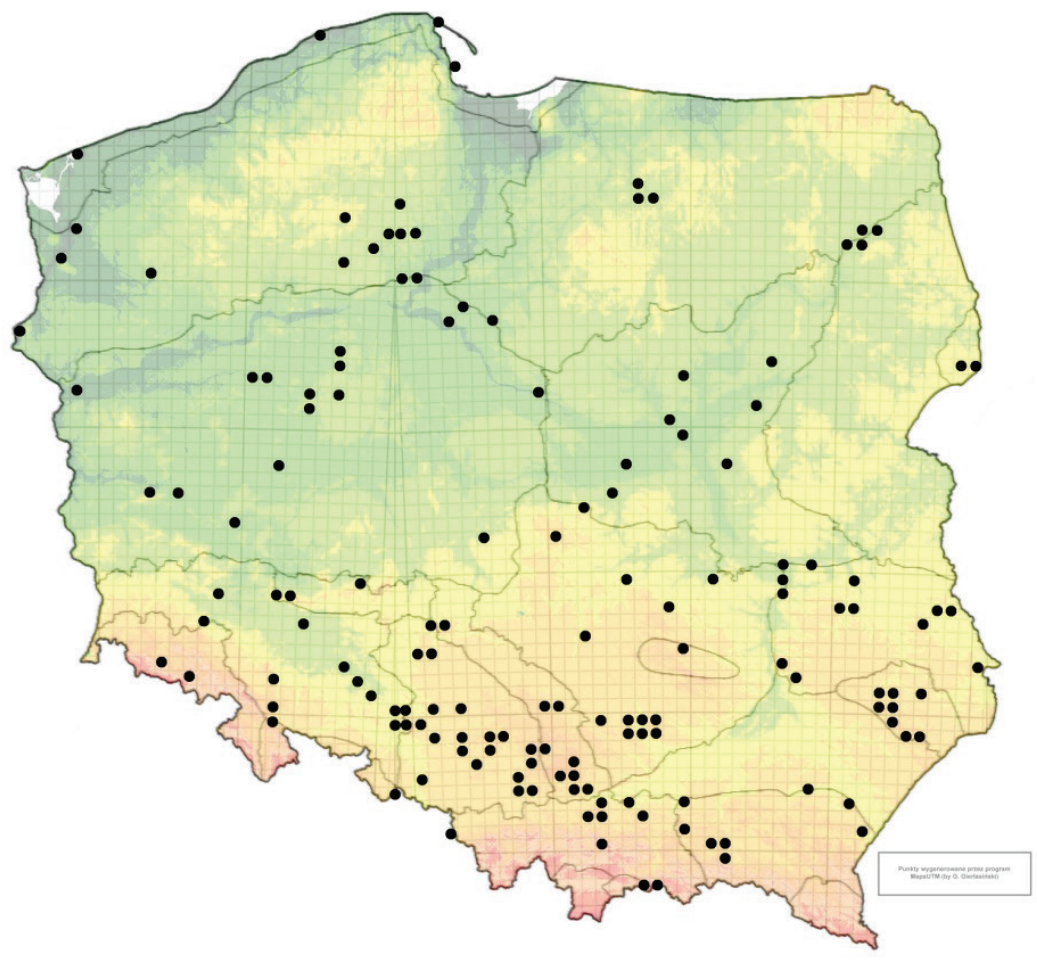

Fig. 1. Neides tipularius - distribution in Poland.

Lower Silesia: *Bardo [XR29] - 25 May 1932, 1 ex. leg. F. Kirsch (USMB); *Brzeg [XS73] - 1 ex., 26 Feb 2011, leg. J. Regner; Gogolin [BA89] - Lis J.A. 1989, Hebda 2006; *12 Sep 1928, 2 exx., 16 Oct 1930, 2 exx., leg. H. Nowotny (USMB); Górażdże [BB80] Hohol-Kilinkiewicz \& Czaja 2006; *Ochodze [XS91] - 6 Sep 2004, 1 ex., leg. E. Fąferko (DBUO); Kamień Śląski [BB90] - Hebda 2006; *Ligota Wielka [XS22] - 20 Apr 1930, 1 ex., leg. E. Drescher (USMB); *Lipowa [XS82] - 31 Jul 2004, 2 exx., 20 Aug 2004, 1 ex., 19 Sep 2004, 2 exx., leg. E. Fąferko (DBUO); *Raszowa n. Lubin [WS88] - 4 Aug 1928, 2 exx. (DBUO); Winnica [WS76] - Assmann 1854; Wrocław [XS46] - Assmann 1854; Wrocław, Karłowice [XS46] - Assmann 1854; *Ząbkowice Śląskie [XS20] - 9 Feb 1938, 1 ex. leg. H. Nowotny (USMB). 
Lubelska Upland: Bochotnica [EB68] - Cmoluchowa 1964; Felin [FB17] - Ziarkiewicz 1962; Gołąb [EC60] - Cmoluchowa 1971; Gródek [GB03] - Strawiński 1959b; Kazimierz Dolny [EB68] - Cmoluchowa 1964; Lublin [FB07] - Ziarkiewicz 1957; Michałówka [EB69] Strawiński 1957b; Opoka [EB63] - Cmoluchowa 1971; Podgórz [EB68] - Cmoluchowa 1964; Bagno Serebryskie Res. [FB77] - Lechowski \& Smardzewska-Gruszczak 2004; Brzeźno Res. [FB87] - Smardzewska-Gruszczak \& Lechowski 2006; Ruda Czechowska [EB69] Strawiński 1963; Wandzin [FB19] - Strawiński 1956c, Strawiński 1957a, Ziarkiewicz 1958; Wolica [EC80] - Smreczyński 1954; Wólka Gołębska [EC60] - Strawiński 1963; Zaklików [EB72] - Cmoluchowa 1971; Zawadówka Res. [FB66] - Smardzewska-Gruszczak \& Lechowski 2000; Zemborzyce [FB07] - Cmoluchowa 1958.

Małopolska Upland: *Bogucice [DA79] - 3 Aug 1999, 4 exx., 7 Aug 1999, 1 ex., 10 Aug 1999, 2 exx., leg. B. \& J.A. Lis (DBUO); *Grabowiec Res. n. Pińczów [DA57] 17 Jul 1954, 1 ex., leg. S. Nowakowski (ZMPA); Kruszewiec [DB59] - Strawiński 1936; *Krzyżanowice Dolne [DA68] - 16 Sep 1954, 5 exx., leg. S. Nowakowski (ZMPA), 6 Aug 2000, 1 ex., leg. B. \& J. A. Lis (DBUO); Leszczyny [DB87] - Strawiński 1936; Sielec [DA39] - Fedorko 1959; *Polana Polichno Res. [DA69] - 28 May 1959, 1 ex., leg. W. Bazyluk (ZMPA); *Pińczów [DA69] - 5 Aug 1998, 1 ex., KNB (DBUO); *Skorocice [DA78], 10 Aug 1999, 1 ex. leg. B. \& J. A. Lis (DBUO); Winiary [DA78] - 26 Aug 1954, 1 ex., leg. S. Nowakowski (ZMPA).

Mazowiecka Lowland: Dziekanów Leśny [DC99] - Bilewicz-Pawińska 1965; *Gaj Policzko [DB25] - 1 Aug 2007, 1 ex. leg. W. Żyła (USMB); Jadwisin [ED41] - BilewiczPawińska 1965; *Lomna [DD80] - 17 Oct 1980, 1 ex., 26 Apr 1982, 1 ex., leg. A. Kędziorek (ZMPA); Otwock [EC27] - Smreczyński 1954; Puszcza Biała forest [ED54] - Cmoluchowa \& Lechowski 1993; Radom [EB19] - Tomasik 2014; *Romanów [DC02] - 18 Aug 2011, 1 ex., leg. J. Kalisiak; *Rogów [DC24] - 3 exx., 6 Sep 2014, leg. M. Bunalski; Skierniewice [DC45] - Strawiński 1936; Szymanów [DC57] - Smreczyński 1954; Warszawa, Młociny [DC99] Bilewicz-Pawińska 1961, Bilewicz-Pawińska 1965; Zbroszki [DD93] - Lechowski 1989.

Mazurian Lake District: general - Mikołajski 1962b; Klewki [DE75] - Mikołajski 1961; Kujawy [CD67] - Smreczyński 1954; Olsztyn, Posorty [DE65] - Mikołajski 1962d; Redykajny Res. [DE66] - Mikołajski 1962a; Tomaszkowo [DE65] - Mikołajski 1961.

Pieniny Mts: Kras [DV67] - Taszakowski \& Pasińska 2017.

Podlasie Lowland: Dawidowizna [FE12] - Lis J. A. et al. 1995; Downary Plac [FE12] - Lis J. A. et al. 1995; Goniądz [FE12] - Lis J. A. et al. 1995; Osowiec [FE02] - Lis J. A. et al. 1995; Osowiec, Twierdza [FE02] - Lis J. A. et al. 1995; Czerwone Bagno Res. [FE13] - Lis J. A. et al. 1995; Układek [FE13] - Lis J. A. et al. 1995; Wólka Piaseczna [FE13] - Lis J. A. et al. 1995; Wroceń [FE23] - Lis J. A. et al. 1995.

Pomeranian Lake District: Bielinek over the Odra River Res. [VU46] - Engel \& Hedicke 1934, Engel 1938; Bory Tucholskie forest [CE05] - Kuhlgatz 1901, Cmoluchowa \& Lechowski 1993; Cisiny [CE13] - Kosicki 1958; Dobrcz [CE00] - Wrzesińska et al. 2013; Goleniów [VV83] - Wagner 1941; *Gołąbek n. Tuchola [XV64] - 19 Aug 2004, 1 ex., leg. B. \& J. A. Lis (DBUO); *Gostycyn [XV82] - 17 Aug 2004, leg. B. \& J. A. Lis (DBUO); Recz [WV30] - Karl 1935; *Piła n. Gostycyn [XV82] - 11 Aug 2004, 2 exx., 17 Aug 2004, 3 exx., leg. B. \& J. A. Lis (DBUO); *Płazowo [XV93] - 9 Aug 2004, 2 exx., leg. B. \& J. A. Lis (DBUO); Szczecin [VV71] - Schmidt 1928; Wierzchlas Res. [CE03] - Kosicki 1958; Zakrzewska Osada [XV61] - Tarnawski 2013; *Zamrzenica [XV82] - 8 Aug 2004, 7 exx., 12 Aug 2004, 2 exx., leg. B. \& J. A. Lis (DBUO);

Roztocze Upland: Górecko Stare [FA49] - Cmoluchowa \& Lechowski 1994; Kosobudy [FB41] - Strawiński 1956a; Łabunie [FB61] - Strawiński 1960; Paary [FA68] - Strawiński 1956a; Bukowa Góra Res. [FB30] - Strawiński 1956a, Strawiński 1964, Lechowski \& 
Cmoluchowa 1993, Cmoluchowa \& Lechowski 1994; Nart Res. [FB40] - Strawiński 1956a; Tartaczna Góra Res. [FB30] - Strawiński 1966b; Susiec [FA58] - Strawiński 1959a; Turzyniec [FB31] - Strawiński 1956a; Wólka Łosiniecka [FA68] - Strawiński 1959a; Zwierzyniec [FB30] - Strawiński 1956a, Strawiński 1966a.

Sandomierska Lowland: Kłaj [DA53] - Stobiecki 1915; Łańcut [EA84] - Nowicki 1868; Tarnów [DA93] - Smreczyński 1954.

Świętokrzyskie Mts: Psarska Mt. [DB94] - Strawiński 1962.

Trzebnickie Hills: Kuraszków [XS38], Maczów [XS38] - Bugaj-Nawrocka et al. 2018; Oborniki Śląskie [XS38] - Polentz 1943, Bugaj-Nawrocka et al. 2018; Ose [XS89] - Lanzke \& Polentz 1942; Rościsławice [XS28] - Bugaj-Nawrocka et al. 2018.

Upper Silesia: *Bażany [CB04] - 20 Sep 1936, 1 ex. leg. H. Nowotny (USMB); Bobrek [CA74] - Stobiecki 1886, Lis J. A. 1989, Lis J. A. \& Lis B. 1998; Bukowno, Pustynia Starczynowska [CA87] - Lis J. A. 1989, Lis J. A. \& Lis B. 1998; Bytom [CA58] - Lis J. A. 1989, Lis J. A. \& Lis B. 1998; Chełmek [CA75] - Stobiecki 1886, Stobiecki 1915, Lis J. A. 1989, Lis J. A. \& Lis B. 1998; Dąb [CA75] - Stobiecki 1886, Lis J. A. 1989, Lis J. A. \& Lis B. 1998; Dolina Żabnika Res. [CA86] - Chłond et al. 2005; Gliwice [CA37] - Lis J. A. 1989, Lis J. A. \& Lis B. 1998; Jemielnica [CB10] - Lis J. A. 1989, Lis J. A. \& Lis B. 1998, 19 Oct 1936, 2 exx., leg. H. Nowotny (USMB); Libiąż [CA75] - Stobiecki 1886, Lis J. A. 1989, Lis J. A. \& Lis B. 1998; Lipowiec [CA84] - Stobiecki 1886, Lis J. A. 1989, Lis J. A. \& Lis B. 1998; Łabędy [CA37] - Lis J. A. 1989; *Lężczok Res. [CA05] - 24 Jul 2006, 1 ex., 1 Sep 2006, 1 ex., 11 Sep 2006, 1 ex., 16 Sep 2006, 1 ex., leg. T. Masarczyk (DZUS); *Pławy [CA68] - 20 Jul 2008, 1 ex., leg. K. Rydzoń; St. Anne Mt. LP [BA99] - Lis J. A. 1989, Lis J. A. \& Lis B. 1998, Lis B. \& Danielczok-Demska 2001; Potępa [CB30] - Lis J. A. 1989, Lis J. A. \& Lis B. 1998; *Ruda Śląska [CA46] - 09 Aug 2008, 1 ex., 15 Aug 2008, 1 ex., 20 Aug 2008, 3 exx., 17 Oct 2008, 1 ex., leg. L. Jezuit (DZUS); *Rudziniec [CA18] - 8 Jul 2015, 1 ex., at light, leg. R. Dobosz (USMB); *Strzelce Opolskie [CA09] - 17 Jul 2005, 1 ex., leg. M. Hamal (DBUO); Szymiszów [CA09] - Lis J. A. 1989, Lis J. A. \& Lis B. 1998; *Wojciechów [CB14] - 10 Aug 1938, 1 ex., leg. H. Nowotny (USMB); Zbrosławice [CA38] Lis J. A. 1989, Lis J. A. \& Lis B. 1998; Żabie Doły Res. [CA57] - Musik 2010; Żarki [CA84] - Stobiecki 1886, Lis J. A. 1989, Lis J. A. \& Lis B. 1998.

Western Beskidy Mts: Bieńkowice [DA32] - Smreczyński 1906b; *Cieszyn [CA21] - 12 Aug 2006, 1 ex., leg J. Spandel (DBUO), 3 exx. (USMB); Kasina Wielka [DA30] Smreczyński 1910; Krzyszkowice [DA22] - Smreczyński 1906b; Melsztyn [DA62] Smreczyński 1906a, Smreczyński 1954; Piwniczna [DV77] - Smreczyński 1954; Wieliczka [DA33] - Stobiecki 1886.

Western Sudetes Mts: Cieplice Śląskie Zdrój [WS43] - Assmann 1854; Sołtys Mt. [WS62] - Assmann 1854.

Wielkopolsko-Kujawska Lowland: Brudzyń [XU65] - Szulczewski 1913; Czarnowska Górka ad Czarnów [VU82] - Hebda \& Rutkowski 2015; *Dolina Samy val. [XU03] - 1 ex., 16 May 2009, 2 exx., 11 Aug 2010, 1 ex., 10 Jul 2011, leg. M. Bunalski; Duninów [CD92] Strawiński 1965; *Dziekanowice [XU62] - 1 ex., 9 May 2010, leg. M. Bunalski; Janowiec Wielkopolski [XU64] - Szulczewski 1908; *Jaryszewo, valley of the Warta river [XU13] 1 ex., 27 Jul 2014, 1 ex., 20 Jul 2015, leg. M. Bunalski; *Jezioro Sycyńskie lake [XU03] 1 ex., 11 Aug 2015, leg. M. Bunalski; Kromolin [CC52] - Strawiński 1936; Milsko [WT55] 1 ex., 9 Sep 2017, leg. R. Orzechowski; Papowo Toruńskie [CD48] - Cmoluch 1960; Puszcza Zielonka forest [XU42] - Skórka 1994; Suponin [CE10] - Wrzesińska et al. 2013; *Sycyn Dolny [XU03] - 2 exx., 27-30 Apr 2009, 5 exx., 20 Jun 2009, 1 ex., 17 Jun 2009, 1 ex., 10 Aug 2009, 10 exx., 19 Jul 2010, 6 exx., 25 Jul 2010, 3 exx., 29 Apr 2011, 2 exx., 20 May 2011, 2 exx., 17 Jul 2011, 1 ex., 16 Jul 2011, 1 ex., 3 Aug 2011, 2 exx., 3 Aug 2011, 1 ex., 
7 Jul 2012, 2 exx., 7 Aug 2012, 1 ex., 7 Jul 2012, 2 exx., 28 Aug 2012, 3 exx., 29 Aug 2012, 1 ex., 1 May 2013, 1 ex., 23 Aug 2013, 1 ex., 7 Sep 2013, leg. M. Bunalski; *Sycyn Dolny [XU13] - 1 ex., 4 Jun 2010, 3 exx., 2 Aug 2012, 1 ex., 11 Aug 2013, leg. M. Bunalski; Toruń [CD37] - Smreczyński 1954; Turew [XT27] - Trojan 1989; *Uroczysko Maruszka [XU41] 1 ex., 12 Aug 2009, leg. M. Bunalski; *Wschowa [WT93] - 1 ex., 4 Mar 2014, leg. R. Matuszczak; Zielona Góra [WT35] - Gruhl 1929; 1 ex., 6 May 2014, leg. R. Orzechowski.

Berytinini Southwood \& Leston, 1959

\section{Berytinus (Berytinus) clavipes (Fabricius, 1775)}

Berytus clavipes: Gravenhorts 1836: 81, Siebold 1839: 433; Scholtz 1847: 151; Łomnicki 1882: 43; Stobiecki 1886: 133; Kotula 1890: 136; Smreczyński 1906b: 53, 1910: 111; Scholz 1931: 155. Beritinus clavipes: Strawiński 1959b: 145, 1960: 152, 1963: 22.

Range element: Pacific-Atlantic.

Bionomics: Mesophilous; oligophagous, mainly on Ononis spp.; one generation (possible two) a year.

Remarks: Common and widely distributed in Poland.

Distribution in Poland (Fig. 2).

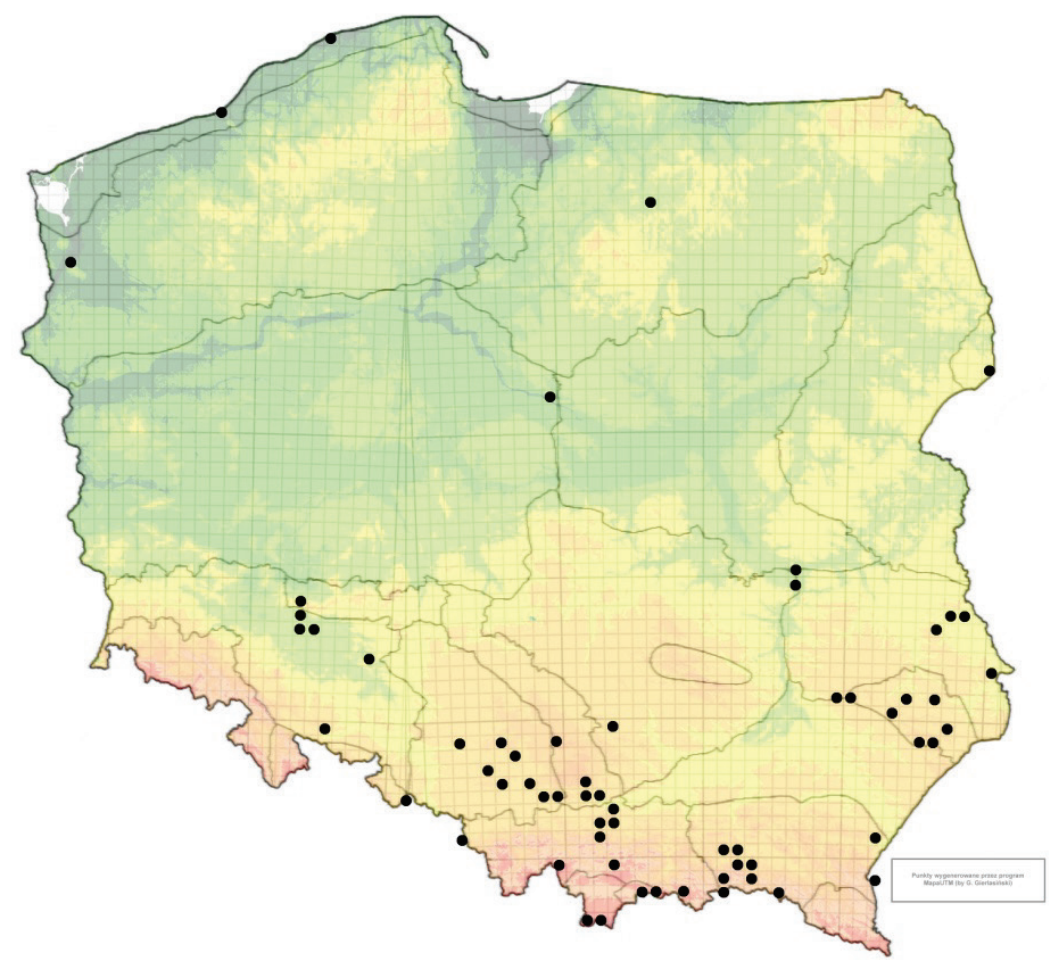

Fig. 2. Berytinus clavipes - distribution in Poland.

Baltic Coast: *Czajcze n. Mielno [WA71] - 18 Aug 1996, 2 exx., leg. B. \& J. A. Lis (DBUO); Słowiński NP [XA46] - Korcz 2003.

Białowieża Forest: Białowieża [FD94] - Strawiński 1956b. 
Eastern Beskidy Mts: Bednarka [EA20, EV29], Blechnarka [EV17], Dobrynia [EV39], Gładyszów [EV18], Jaśliska [EV57], Krempna [EV38] - Taszakowski \& Gorczyca 2018; Krościenko [FV28] - Smreczyński 1906b; Libusza [EA10], Lipinki [EA20] - Taszakowski \& Gorczyca 2018; Przemyśl [FA21] - Kotula 1890; Wysowa-Zdrój [EV17] - Taszakowski \& Gorczyca 2018.

Eastern Sudetes Mts: *Gipsowa Góra Res. [YR14] - 8 Sep 1932, 10 exx., leg. H. Nowotny (USMB).

Krakowsko-Wieluńska Upland: Kraków, Błonia [DA24] - Stobiecki 1886; Kraków, Dębniki [DA14] - Stobiecki 1886; Kraków, Krzemionki [DA14] - Smreczyński 1906b; Kraków, Panieńskie Skały [DA14] - Stobiecki 1886; Kraków, Sikornik [DA14] - Stobiecki 1886; *Olsztyn [CA98] - 1 Aug 1999, 2 exx, 26 Jan 1999, 1 ex., leg. A. Pańczyk (DBUO); Zabierzów [DA15] - Stobiecki 1886.

Lower Silesia: Kopalina [XS84] - Scholz 1931; Śliwice [XR59] - Assmann 1854; Wrocław, Karłowice [XS46] - Scholtz 1847, Assmann 1854, Scholz 1931; Wrocław, Popowice [XS36] - Assmann 1854, Scholz 1931; Wrocław, Rędzin [XS37] - Scholz 1931; Wrocław, Swojczyce [XS46] - Scholtz 1847, Assmann 1854.

Lubelska Upland: Gródek [GB03] - Strawiński 1959b; Janów Lubelski [EB91] - Piasecka 1960; Michałówka [EB69] - Strawiński 1957b; Bagno Serebryskie Res. [FB77] - Lechowski \& Smardzewska-Gruszczak 2004; Brzeźno Res. [FB87] - Smardzewska-Gruszczak \& Lechowski 2006; Zawadówka Res. [FB66] - Smardzewska-Gruszczak \& Lechowski 2000; Ruda Czechowska [EB69] - Strawiński 1963; Wólka Gołębska [EC60] - Strawiński 1963.

Małopolska Upland: Sielec [DA39] - Fedorko 1959.

Mazurian Lake District: Olsztyn, Posorty [DE65] - Mikołajski 1962c, 1962d.

Pieniny Mts: Biała Skała [DV57] - Hebda \& Ścibior 2016; Kras [DV67] - Taszakowski \& Pasińska 2017; Krościenko by the Dunajec River [DV57] - Smreczyński 1954; Pieniny, general [DV57] - Smreczyński 1954, Taszakowski \& Pasińska 2017.

Pomeranian Lake District: Szczecin [VV71] - Dohrn 1860.

Roztocze Upland: Biała Góra n. Tomaszów Lubelski [FA79] - Cmoluchowa \& Lechowski 1994; Kosobudy [FB41] - Strawiński 1956a; Łabunie [FB61] - Strawiński 1960; Tartaczna Góra Res. [FB30], Strawiński 1966b; Susiec [FA58] - Strawiński 1959a; Wólka Łosiniecka [FA68] - Strawiński 1959a; Zwierzyniec [FB30] - Strawiński 1956a.

Sandomierska Lowland: Szklarnia Res. [FB01] - Lechowski \& Smardzewska-Gruszczak 1998.

Tatry Mts: Kościeliska val. [DV15] - Smreczyński 1954, Lis B. et al. 2004; Strążyska val. [DV25] - Smreczyński 1954.

Trzebnickie Hills: Oborniki Śląskie [XS38] - Polentz 1943.

Upper Silesia: *Będzin [CA67] - 25 Jun 2008, 1 ex., leg. A. Kulis (DZUS); Bycina [CA28] - Stobiecki 1886, Lis J. A. 1989, Lis J. A. \& Lis B. 1998; Chełmek [CA75] - Stobiecki 1886, Lis J. A. 1989, Lis J. A. \& Lis B. 1998; Dąb [CA75] - Stobiecki 1886, Lis J. A. 1989, Lis J. A. \& Lis B. 1998; Lipowiec [CA84] - Stobiecki 1886, Lis J. A. 1989, Lis J. A. \& Lis B. 1998; *Piekary Śląskie [CA58] - 23 May 2008, 1 ex., leg. M. Sikora (DZUS); Regulice [CA94] Smreczyński 1954; *Ruda Śląska [CA46] - 9 Aug 2008, 1 ex., leg. L. Jezuit (DZUS); *Tychy [CA55] - 26 Mar 2007, 2 exx., leg. D. Nawara (DZUS); Żarki [CA84] - Stobiecki 1886, Lis J. A. 1989, Lis J. A. \& Lis B. 1998.

Western Beskidy Mts: Babia Góra Mt. [CV99] - Lis B. et al. 2002, Celary 2003; Bieńkowice [DA32] - Smreczyński 1906b; Chełm n. Myślenice [DA21] - Smreczyński 1954; *Cieszyn [CA21] - 1 ex. (DZUS); Krzyszkowice [DA22] - Smreczyński 1906b; Łomnica [DV87] - Smreczyński 1954; Niedźwiedź [DV39] - Smreczyński 1910; Wieliczka [DA33] Stobiecki 1886.

Wielkopolsko-Kujawska Lowland: Duninów [CD92] - Strawiński 1965. 
"Prussia" - Siebold 1839.

"Silesia" - Gravenhorts 1836, Schummel 1836.

"Western Galicja" - Łomnicki 1882.

\section{Berytinus (Berytinus) hirticornis hirticornis (Brullé, 1836)}

Berytus hirticornis: Smreczyński 1906b: 53, 1908: 71; Scholz 1931: 155.

Berytus trichocerus: Scholtz 1847: 151.

Range element (for all subspecies together): Kirghisian-Atlantic.

Bionomics: Mesophilous/xerotermophilous; polyphagous; one generation a year.

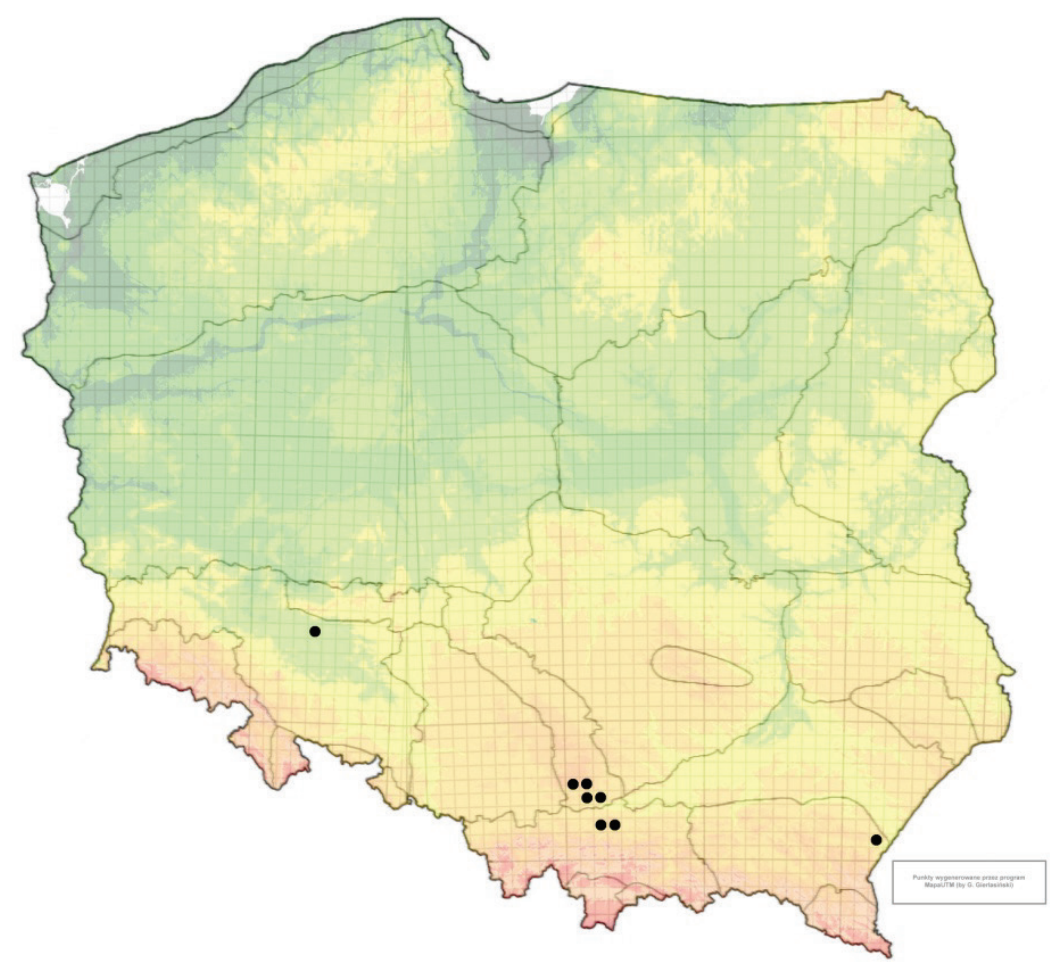

Fig. 3. Berytinus hirticornis - distribution in Poland.

Remarks: Very rare in Poland.

Identification of the specimens (deposited in the Museum of Natural History ISEA PAS in Kraków) from Rząska (Krakowsko-Wieluńska Upland) and from Przemyśl (Western Beskidy Mts), was verified and confirmed.

This species is known only from very old records in our country, moreover it is a very rare species in the territory of Slovakia and unknown in Moravia (Stehlík \& Vavř́nová 1995), therefore its occurrence in Poland needs confirmation.

Distribution in Poland (Fig. 3):

Eastern Beskidy Mts: Przemyśl [FA21] - Smreczyński 1908.

Krakowsko-Wieluńska Upland: Czerna [DA05] - Smreczyński 1906b; Kraków, Borek Fałęcki [DA24] - Smreczyński 1906b; Kraków, Krzemionki [DA14] - Smreczyński 1906b; 
Kraków, Nowa Wieś [DA24] - Smreczyński 1906b; Rząska [DA15] - Smreczyński 1906b; Sowiarka [DA05] - Smreczyński 1906b.

Lower Silesia: Wrocław [XS46] - Scholtz 1847, Assmann 1854, Schumacher 1912, Scholz 1931.

Western Beskidy Mts: Bieńkowice [DA32] - Smreczyński 1906b; Dobczyce [DA32] Smreczyński 1906b; Krzyszkowice [DA22] - Smreczyński 1906b.

\section{Berytinus (Berytinus) minor minor (Herrich-Schäffer, 1835)}

Berytus commutatus: Nowicki 1870: 238.

Berytus minor: Scholtz 1847: 151; Łomnicki 1882: 43; Kotula 1890: 136; Smreczyński 1906b: 53, 1910: 111; Stobiecki 1915: 147; Tenenbaum 1921: 4; Scholz 1931: 155;

Beritinus minor: Strawiński 1960: 152, 1962: 184;

Berytus montivagus: Smreczyński 1906b: 53.

Range element (for all subspecies together): Siberian-Atlantic.

Bionomics: Mesophilous; mainly Trifolium spp.; one (possible two) generation a year.

Remarks: Common and widely distributed in Poland.

Distribution in Poland (Fig. 4):

Bieszczady Mts: Stuposiany [FV24] - Cmoluchowa \& Lechowski 1977.

Eastern Beskidy Mts: Bednarka [EA20, EV29], Dobrynia [EV39] - Taszakowski \& Gorczyca 2018; upper Ropa River val. [EV17] - Taszakowski 2012; Hureczko [FA31] Kotula 1890; Iwonicz [EV59] - Strawiński 1953; Libusza [EA10], Lipinki [EA20] Taszakowski \& Gorczyca 2018; Prałkowce [FA21] - Kotula 1890; Przemyśl, Lipowica [FA21] - Kotula 1890.

Eastern Sudetes Mts: *Gipsowa Góra Res. [YR14] - 8 Sep 1932, 2 exx., leg. H. Nowotny (USMB); *Masyw Śnieżnika Mts. [XR46] - 24 Aug 2006, 1 ex., KNB (DBUO).

Krakowsko-Wieluńska Upland: Kraków, Bielany [DA14] - Smreczyński 1906b; Kraków, Przylasek Rusiecki [DA34], Modlniczka [DA15] - Stobiecki 1915; Sąspowska val. [DA16] Chłond \& Gorczyca 2009.

Lower Silesia: *Kwietno [XS06] - 8 May 1944, 5 exx., 27 May 1944, 1 ex., leg. A. Lanzke (ZMPA); *Leszczyna n. Legnica [WS65] - 14 May 1932, 1 ex. (DBUO); *Szymanów [XS25] - 29 Jul 1943, 1 ex., leg. A. Lanzke (ZMPA); *Ujazd Dolny [XS06] - 18 Aug 1944, 1 ex., leg.

A. Lanzke (ZMPA); Wrocław [XS46] - Scholtz 1847, Assmann 1854, Scholz 1931.

Małopolska Upland: *Krzyżanowice Dolne [DA68] - 16 Sep 1954, 1 ex., 19 Sep 1954, 1 ex., leg. S. Nowakowski (ZMPA).

Lubelska Upland: Bystrzyca [FB18] - Lechowski 1984; Dratów [FB39] - Cmoluchowa \& Lechowski 1988; Kaniwola [FB49] - Cmoluchowa \& Lechowski 1988; Lublin, Botanical Garden [FB08] - Cmoluchowa 1960; Łuszczów [FB28] - Lechowski 1984; Podgórz [EB68] Cmoluchowa 1964; Brzeźno Res. [FB87] - Smardzewska-Gruszczak \& Lechowski 2006; Zawadówka Res. [FB66] - Smardzewska-Gruszczak \& Lechowski 2000; Rudnik [FB18] Lechowski 1984; Turka [FB18] - Lechowski 1984; Wandzin [FB19] - Strawiński 1956c; Wrotków [FB07] - Cmoluchowa 1958; Zemborzyce [FB07] - Cmoluchowa 1958.

Mazowiecka Lowland: Chylice [EC06] - Lechowski 1989; Klembów [ED20] - Lechowski 1989; Warszawa [EC08] - Hałka-Wojciechowicz 1996.

Pieniny Mts: general [DV57] - Taszakowski \& Pasińska 2017.

Pomeranian Lake District: Gwda Wielka [XV15] - Burdajewicz \& Nowacka 1995; *Piła n. Gostycyn [XV82] - 11 Aug 2004, 1 ex., leg. B. \& J. A. Lis (DBUO); Słupsk [XA33] - Karl 1935; *Szczecin [WT82] - 26 May 1915, 1 ex., leg. P. Noack (ZMPA). 
Roztocze Upland: Łabunie [FB61] - Strawiński 1960; Nart Res. [FB40] - Tenenbaum 1921; Ulów [FA69] - Tenenbaum 1921; Zwierzyniec [FB30] - Strawiński 1966a.

Sandomierska Lowland: Lipie [EA69] - Stobiecki 1915.

Świętokrzyskie Mts: Łysa Góra [EB03] - Strawiński 1962; Trzcianka [EB03] - Strawiński 1962.

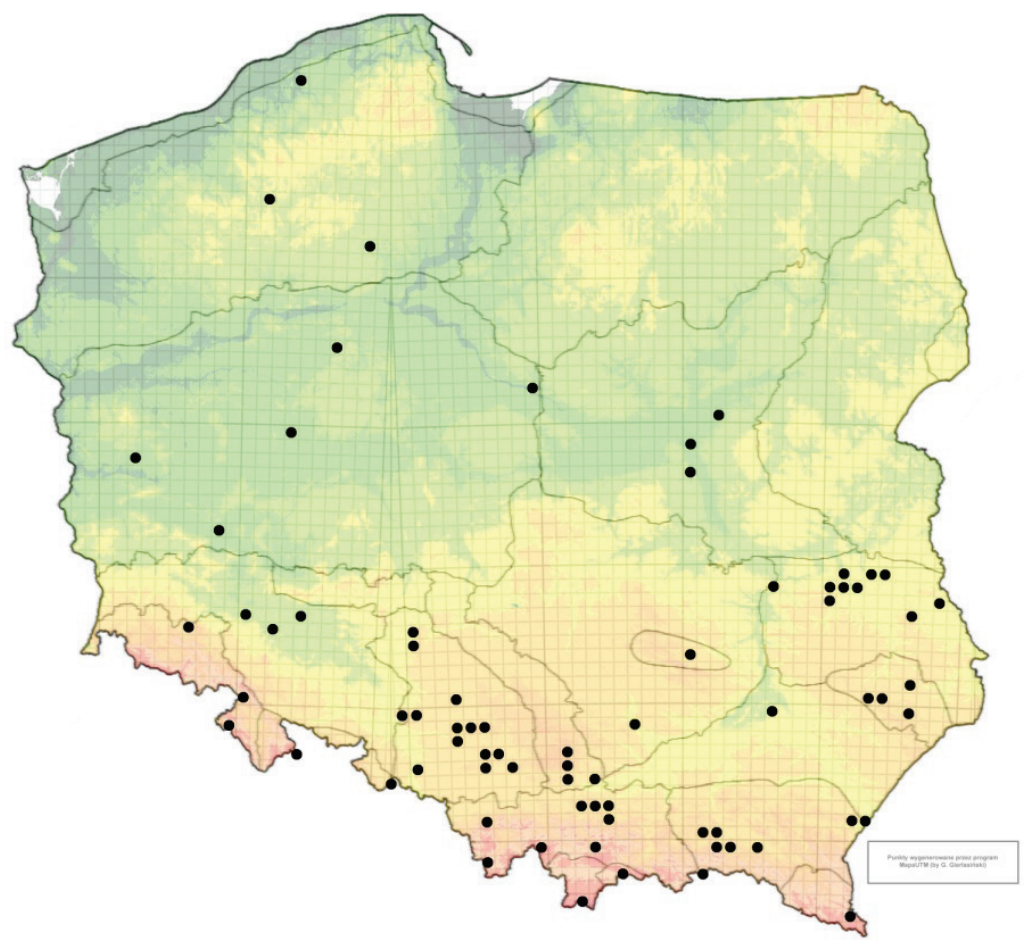

Fig. 4. Berytinus minor - distribution in Poland.

Tatry Mts: general [DV25] - Nowicki 1870, Smreczyński 1954.

Upper Silesia: *Bażany [CB04] - 20 Sep 1936, 1 ex. leg. H. Nowotny (USMB); Bytom [CA58] - Lis J. A. 1989, Lis J. A. \& Lis B. 1998; *Bytom [CA58] - 27 Nov 1931, 1 ex., 4 Aug 1934, 1 ex., 13 Jul 1937, 2 exx., leg. F. Kirsch (USMB); 3 Mar 1934, 4 exx., 5 Oct 1936, 3 exx., 6 Sep 1937, 4 exx., leg. H. Nowotny (USMB); 22 Sep 1934, 1 ex., (ZMPA); *Bytom, Dąbrowa Miejska [CA58] - 14 Nov 1930, 1 ex., 28 Nov 1930, 10 exx., 24 Apr 1931, 10 exx., 16 Sep 1937, 1 ex., leg. H. Nowotny (USMB); Bytom, Stolarzowice [CA48] - Lis J. A. 1989, Lis J. A. \& Lis B. 1998; Chełmek [CA75] - Stobiecki 1915, Lis J.A. 1989, Lis J.A. \& Lis B. 1998; Gliwice [CA37] - Lis J.A. 1989, Lis J.A. \& Lis B. 1998; *Katowice, Podlesie [CA56] - 25 Aug 2009, 1 ex., leg. A. Kempny (DZUS); Segiecki forest [CA48] - Lis J. A. 1989, Lis J. A. \& Lis B. 1998; *Ligota Dolna [CB05] - 21 Sep 1937, 1 ex., 17 Oct 1938, 1 ex., leg. H. Nowotny (USMB); Mikołów, Jamna [CA55] - Lis J. A. 1989, Lis J. A. \& Lis B. 1998; *Mysłowice, Brzezinka [CA66] - 23 May 2009, 1 ex., leg. K. Rydzoń (DZUS); Piekary Śląskie [CA58] - Bugaj-Nawrocka \& Gorczyca 2013; Kamienna Góra Res. [BA99] - Lis B. 1994, Lis J. A. 1989, Lis J. A. \& Lis B. 1998, Lis B. \& Danielczok-Demska 2001; *Łężczok Res. [CA05] - 25 Aug 2006, 3 exx., leg. T. Masarczyk (DZUS), 31 Oct 1937, 3 exx., leg. H. Nowotny (USMB); Szymiszów [CA09] - Lis J. A. 1989, Lis J. A. \& Lis B. 
1998; *Tworóg [CB30] - 23 Oct 1928, 1 ex. leg. H. Nowotny (USMB); *Tychy [CA55] 13 Mar 2007, 2 exx., 24.03.2007, 4 exx., 26 Mar 2007, 5 exx., 29 Mar 2007, 6 exx., 2 Apr 2007, 7 exx., leg. D. Nawara (DZUS); Zbrosławice [CA38] - Lis J. A. 1989, Lis J. A. \& Lis B. 1998.

Western Beskidy Mts: Babia Góra Mt. [CV99] - Lis B. et al. 2002, Celary 2003, *6 Sep 1957, 2 exx., leg. S. Nowakowski (ZMPA); Bieńkowice [DA32] - Smreczyński 1906b; Buczkowice [CA51] - Lis B. \& Dubiel 2013; Dobczyce [DA32] - Smreczyński 1906b; Gruszów [DA42] - Stobiecki 1915; Krzyszkowice [DA22] - Smreczyński 1906b; Niedźwiedź [DV39] - Smreczyński 1910; Słupia [DA41] - Stobiecki 1915; *Sól [CV58] - 6 Aug 2003, 1 ex., leg. J. Ćwikła (DZUS).

Western Sudetes Mts: *Łężyce [WR98] - 22 Jul 2000, 1 ex., leg. M. Widziszewska (DBUO); Nowa Ruda [XS00] - Assmann 1854, Scholz 1931.

Wielkopolsko-Kujawska Lowland: Brudzyń [XU65] - Szulczewski 1913; *Dolina Samy val. [XU03] - 1 ex., 8-10 May 2009 leg. M. Bunalski; Duninów [CD92] - Strawiński 1965; *Głuszyna [XT39] - 1 ex., 20 Jun 1974, leg. A. Korcz; Zawisze [WT27] - 1 ex., 10 Apr 2015, leg. R. Orzechowski.

\section{Berytinus (Lizinus) crassipes (Herrich-Schäffer, 1835)}

Berytus crassipes: Scholtz 1847: 151; Kotula 1890: 136; Smreczyński 1910: 111; Stobiecki 1886: 133, 1915: 147; Schmidt 1928: 195; Scholz 1931: 155.

Range element: Siberian-Atlantic.

Biono mics: Mesophilous; oligophagous (mainly Caryophyllaceae); one generation a year.

Remarks: Common and probably widely distributed in Poland.

Distribution in Poland (Fig. 5):

Baltic Coast: Chłapowo [CF37] - Smreczyński 1954; Gdynia [CF44] - Lis B. \& Kowalczyk 2017.

Eastern Beskidy Mts: Prałkowce [FA21] - Kotula 1890; Wysowa-Zdrój [EV17] Taszakowski \& Gorczyca 2018.

Krakowsko-Wieluńska Upland: Błędowska Desert [CA97] - Lis J. A. 1989, Lis J. A. \& Lis B. 1998; Kraków, Krzemionki [DA14] - Smreczyński 1954; Kraków, Mydlniki [DA24] Stobiecki 1915, Smreczyński 1954. *Olsztyn [CA98] - 19 Jul 1999, 1 ex., leg. A. Pańczyk (DBUO).

Lower Silesia: Kopalina [XS84] - Scholz 1931.

Lubelska Upland: Lublin, Botanical Garden [FB08] - Cmoluchowa 1960; Bagno Serebryskie Res. [FB77] - Lechowski \& Smardzewska-Gruszczak 2004; Zawadówka Res. [FB66] - Smardzewska-Gruszczak \& Lechowski 2000; Turka [FB18] - Lechowski 1984; Wandzin [FB19] - Fedorko 1957; Wrotków [FB07] - Cmoluchowa 1958; Zemborzyce [FB07] - Cmoluchowa 1958.

Małopolska Upland: *Krzyżanowice Dolne [DA68] - 16 Sep 1954, 1 ex., 17 Sep 1954, 1 ex., leg. S. Nowakowski (ZMPA); *Młodzawy [DA68] - 13 Aug 1954, 1 ex., leg. S. Nowakowski (ZMPA).

Masurian Lake District: Ełk [EE86] - Smreczyński 1954.

Pieniny Mts: general [DV57] - Smreczyński 1954, Taszakowski \& Pasińska 2017.

Pomeranian lake District: Szczecin [VV71] - Schmidt 1928.

Roztocze Upland: Bukowa Góra Res. [FB30] - Lechowski \& Cmoluchowa 1993, Cmoluchowa \& Lechowski 1994; Zwierzyniec [FB30] - Strawiński 1956a.

Sandomierska Lowland: Lipie [EA69] - Stobiecki 1915; Zaleszany [EB61] - Stobiecki 1915. 


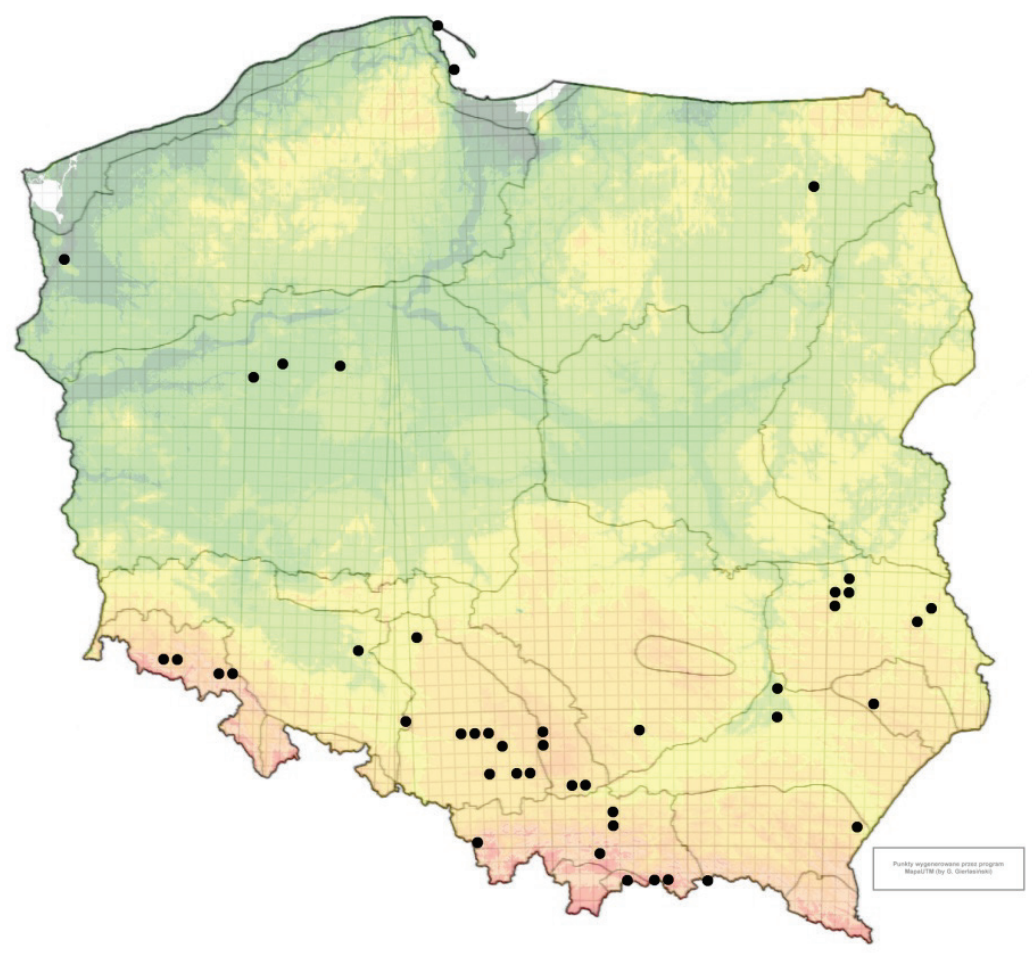

Fig. 5. Berytinus crassipes - distribution in Poland.

Upper Silesia: Balin [CA85] - Stobiecki 1915, Lis J. A. 1989, Lis J. A. \& Lis B. 1998; *Będzin [CA67] - 25 Jun 2008, 1 ex., leg. A. Kulis (DZUS); Bytom [CA58] - Lis J. A. 1989, Lis J. A. \& Lis B. 1998; *Bytom, Stolarzowice [CA48] - 3 Nov 1931, 1 ex., leg. H. Nowotny (USMB); *Bytom [CA58] - 1 Apr 1932, 1 ex. leg. H. Nowotny (USMB), 4 Aug 1934, 1 ex., leg. F. Kirsch (USMB); Chełmek [CA75] - Stobiecki 1886; Lis J. A. 1989, Lis J. A. \& Lis B. 1998; Dąb [CA75] - Stobiecki 1886, Lis J. A. 1989, Lis J. A. \& Lis B. 1998; Segiecki forest [CA48] - Lis J. A. 1989, Lis J. A. \& Lis B. 1998; *Ligota Dolna [CB05] - 17 Oct 1938, 3 exx., leg. H. Nowotny (USMB); St. Anne Mt. LP [BA99] - Lis J. A. 1989, Lis J. A. \& Lis B. 1998, Lis B. \& Danielczok-Demska 2001; Kamienna Góra Res. [BA99] - Polentz 1943, Lis B. 1994, Lis J. A. 1989, Lis J. A. \& Lis B. 1998, Lis B. \& Danielczok-Demska 2001; *Tychy [CA55] - 13 Mar 2007, 1 ex., 26 Mar 2007, 1 ex., 2 Apr 2007, 1 ex., leg. D. Nawara (DZUS); Zbrosławice [CA38] - Lis J. A. 1989, Lis J. A. \& Lis B. 1998.

Western Beskidy Mts: Gruszów [DA42] - Stobiecki 1915; Łomnica [DV87] Smreczyński 1954; Masyw Czantorii Wielkiej Mts. [CA40] - Kędzior et al. 2012; Młodów [DV77] - Stobiecki 1915; Niedźwiedź [DV39] - Smreczyński 1910; Piwniczna [DV77] Smreczyński 1954; Słupia [DA41] - Stobiecki 1915.

Western Sudetes Mts: Cieplice Śląskie Zdrój [WS43] - Scholtz 1847, Assmann 1854, Scholz 1931; Jedlina Zdrój [WS92] - Assmann 1854, Scholz 1931; Staniszów [WS53] Assmann 1854; Szczawno Zdrój [WS82] - Scholtz 1847, Assmann 1854, Scholz 1931.

Wielkopolsko-Kujawska Lowland: *Sycyn Dolny [XU03] - 2 exx., 16 Jul 2011, 1 ex., 7 Aug 2012, leg. M. Bunalski; Wełna [XU24] - Schumacher 1913; Wybranowo [XU64] Schumacher 1913. 
Berytinus (Lizinus) signoreti (Fieber, 1859)

Berytus Signoreti: Smreczyński 1908: 71, 1910: 111;

Berytus montivagus: Kotula 1890: 136.

Beritinus signoreti: Strawiński 1960: 152.

Range element: Pannonian-Atlantic.

Bionomics: Xerothermophilous; oligophagous (mainly Fabaceae); one generation a year.

Remarks: Rare in Poland.

Distribution in Poland (Fig. 6):

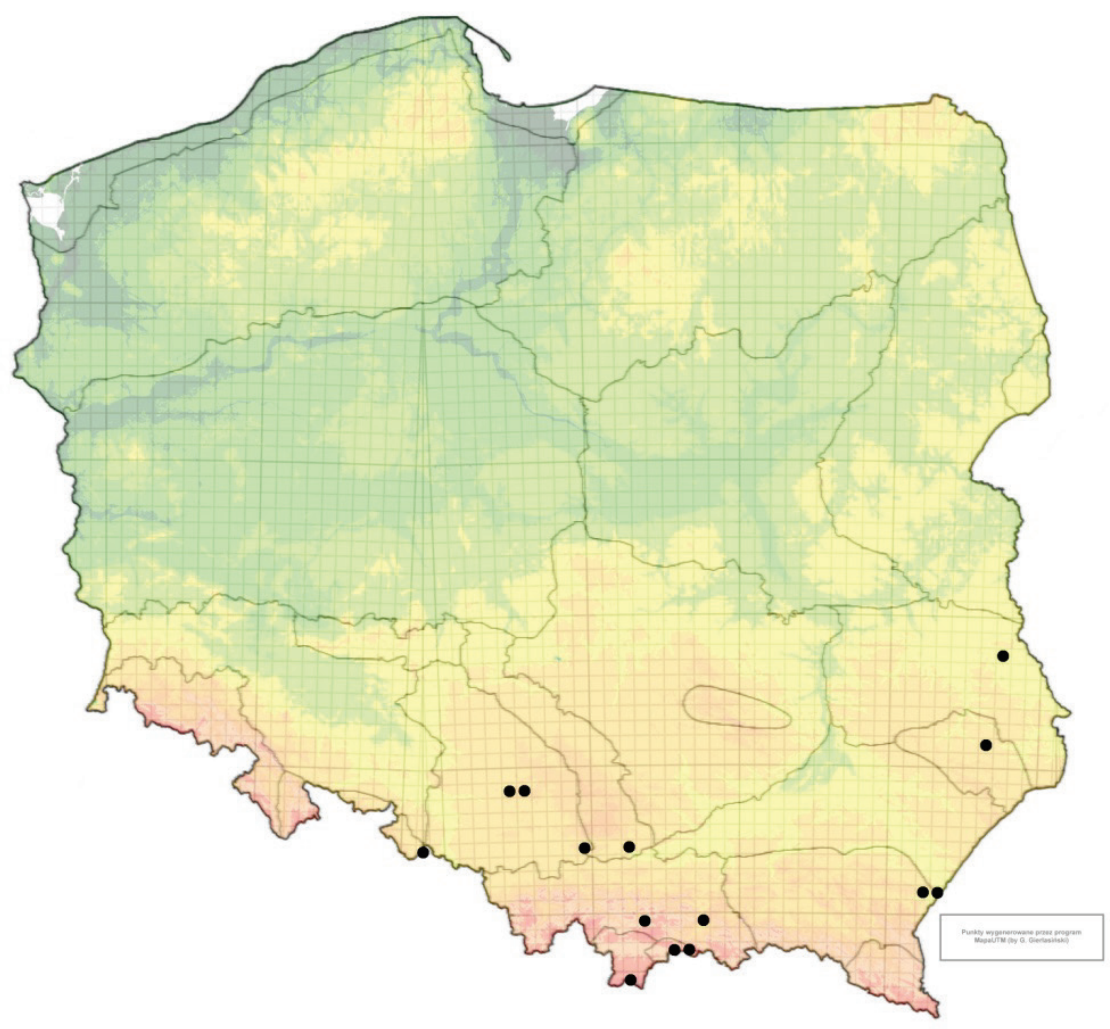

Fig. 6. Berytinus signoreti - distribution in Poland.

Eastern Beskidy Mts: Łuczyce [FA31] - Kotula 1890; Przemyśl [FA21] - Smreczyński 1908; Przemyśl, Wzniesienie [FA21] - Kotula 1890.

Eastern Sudetes Mts: *Gipsowa Góra Res. [YR14] - 8 Sep 1932, 5 exx., leg. H. Nowotny (USMB).

Krakowsko-Wieluńska Upland: Kraków, Borek Fałęcki [DA24] - Smreczyński 1954; Kraków, Mydlniki [DA24] - Smreczyński 1954.

Lubelska Upland: Bagno Serebryskie Res. [FB77] - Lechowski \& SmardzewskaGruszczak 2006a; Polesie Wołyńskie - Lechowski \& Smardzewska-Gruszczak 2006b.

Pieniny Mts: Kras [DV67] - Taszakowski \& Pasińska 2017; Krościenko nad Dunajcem [DV57] - Smreczyński 1954.

Roztocze Upland: Łabunie [FB61] - Strawiński 1960.

Tatry Mts: Strążyska val. [DV25] - Smreczyński 1954. 
Upper Silesia: Bytom [CA58] - Lis J. A. 1989, Lis J. A. \& Lis B. 1998, *Bytom, Dąbrowa Miejska [CA58] - 28 Nov 1930, 1 ex., 24 Apr 1931, 1 ex., leg. H. Nowotny (USMB); *Bytom, Stolarzowice [CA48] - 3 Nov 1931, 1 ex., leg. H. Nowotny (USMB); Regulice [CA94] Smreczyński 1954.

Western Beskidy Mts: Niedźwiedź [DV39] - Smreczyński 1910; Stary Sącz [DV79] Smreczyński 1954.

Gampsocorinae Southwood \& Leston, 1959

Gampsocorini Southwood \& Leston, 1959

Gampsocoris culicinus culicinus Seidenstücker, 1948

Range element (for all subspecies together): Siberian-Mediterranean.

Bionomics: Mesophilous; polyphagous (Lamiaceae, Boraginaceae, Scrophulariaceae, Fabaceae, Rosaceae, Caryophyllaceae and Asteraceae), zoophagy; one generation a year.

Distribution in Poland (Fig. 7):

Eastern Beskidy Mts: Libusza [EA10] - Taszakowski 2015.

Pieniny Mts: Masyw Trzech Koron Mts. [DV57] - Hebda \& Ścibior 2013.

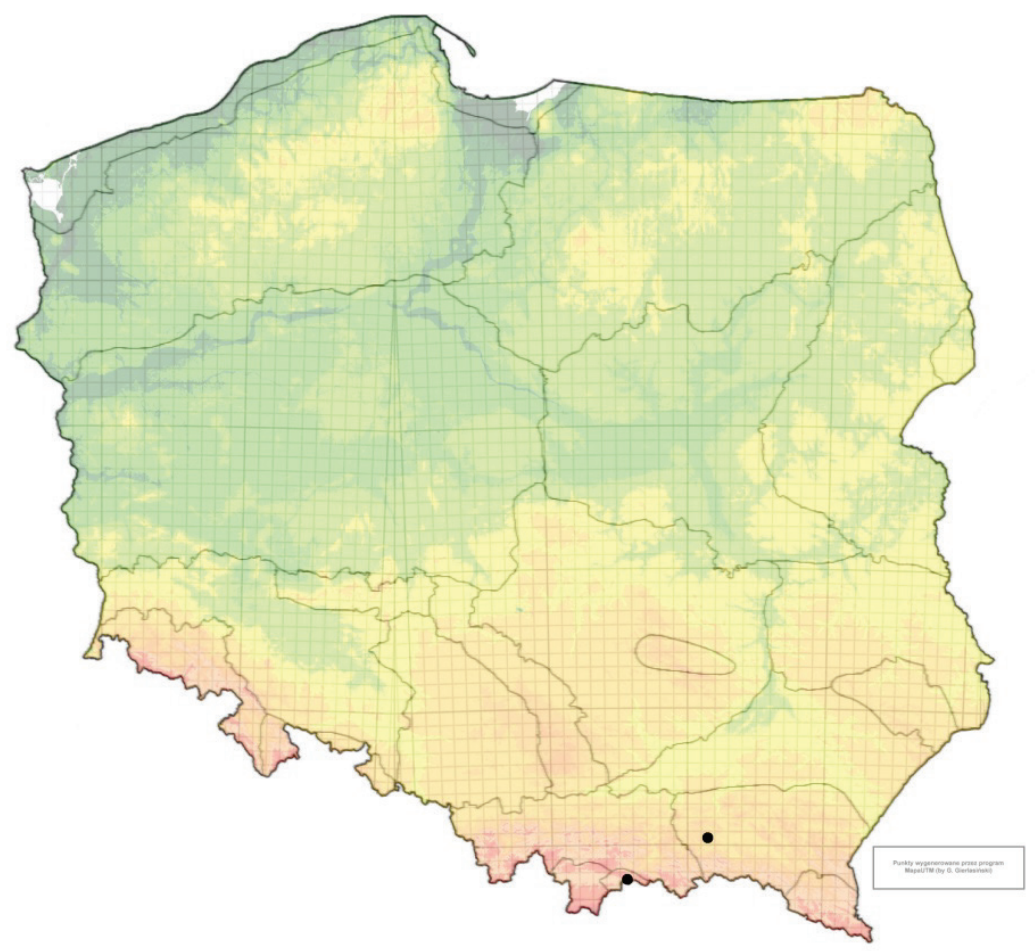

Fig. 7. Gampsocoris culicinus - distribution in Poland. 
Gampsocoris punctipes punctipes (Germar, 1822)

Berytus punctipes: Scholtz 1847: 151.

Metacanthus punctipes: Scholz 1931: 155.

Metacanthus elegans: Smreczyński 1906a: 74, 1908: 71, 1910: 111.

Range element (for all subspecies together): Caspian-Atlantic.

Bionomics: Heliophilous, mesophilous/xerophilous, thermophilous, polyphagous (mainly Ononis spp.; Fabaceae, Boraginaceae, Caryophyllaceae, Rosaceae, Lamiaceae); one or two (or even three) generation a year.

Remarks: All faunistic data on Gampsocoris punctipes and G. culicinus published prior to 1948 (when G. culicinus was described by Seidenstücker) may refer to each of the two mentioned species, and they were presented separately under the Gampsocoris punctipes/culicinus section.

Distribution in Poland (Fig. 8):

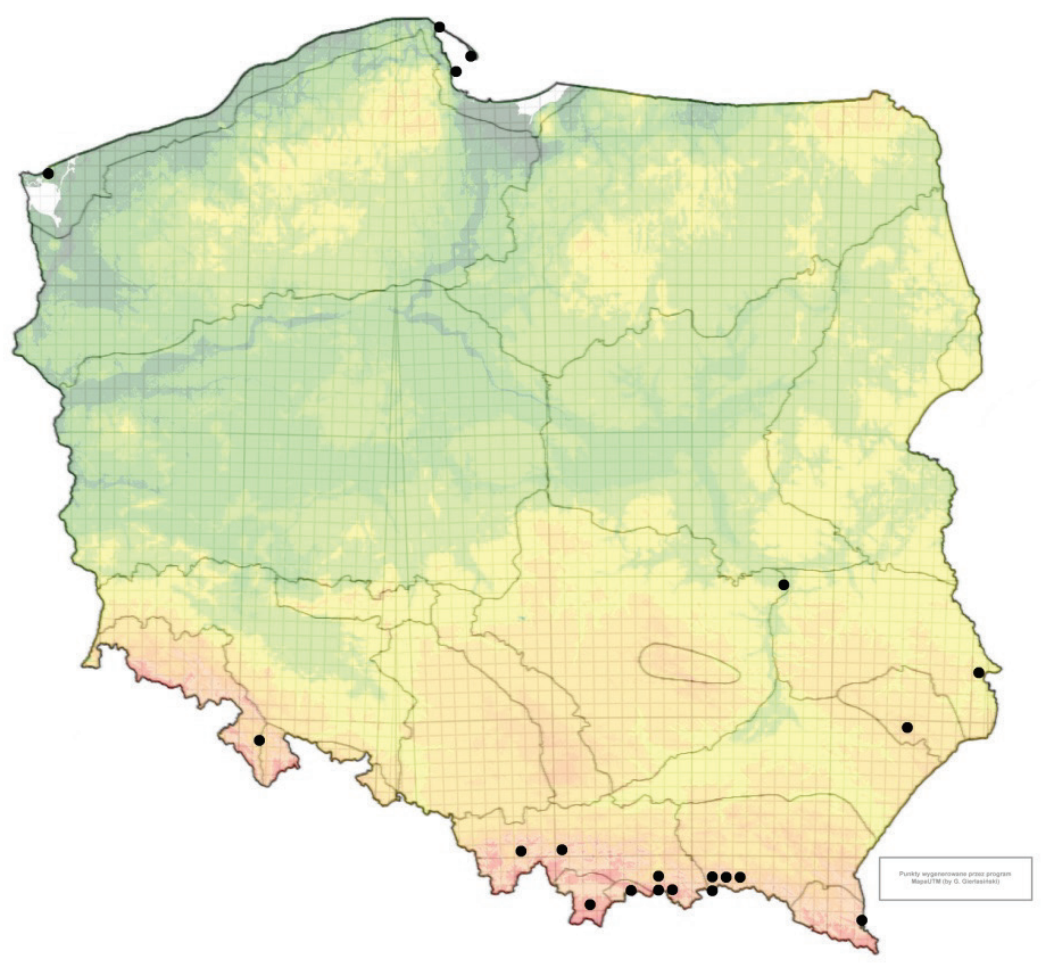

Fig. 8. Gampsocoris punctipes - distribution in Poland.

Baltic Coast: Gdynia [CF44], Wielka Wieś [CF37], Wolin [VV67] - Smreczyński 1954; Hel [CF55] - Łęgowski \& Lis B. 2008.

Bieszczady Mts: general - Gorczyca \& Lis J.A. 2000; Lutowiska [FV25] - Cmoluchowa \& Lechowski 1977.

Eastern Beskidy Mts: *Bartne [EV28] - 7 Aug 2016, 3 exx., leg. A. Taszakowski (DZUS); Blechnarka [EV17], Gładyszów [EV18], Krempna [EV38] - Taszakowski 2015.

Eastern Sudetes Mts: Kłodzko [XR18] - Smreczyński 1954. 
Lubelska Upland: Gródek [GB03] - Strawiński 1959b; Ruda Czechowska [EB69] Strawiński 1963.

Nowotarska Dale: Zakopane [DV26] - Smreczyński 1954.

Pieniny Mts: Krościenko by the Dunajec River [DV57] - Smreczyński 1954; Polana Wyrobek [DV57] - Hebda \& Ścibior 2016.

Roztocze Upland: Hamernia [FA59] - Strawiński 1956a.

Western Beskidy Mts: Łomnica [DV87], Maków Podhalański [DA00] - Smreczyński 1954; *Moszczanica [CA70] - 6 Aug 2003, 1 ex., leg. D. Suchorab (DBUO); Piwniczna [DV77], Rytro [DV78], Żegiestów [DV87] - Smreczyński 1954;

\section{Gampsocoris culicinus/punctipes (data before 1948)}

Distribution in Poland (Fig. 9):

Eastern Beskidy Mts: Przemyśl [FA21], Spława n. Bircza [FA10] - Smreczyński 1908. Eastern Sudetes Mts: Kłodzko [XR18] - Smreczyński 1954.

Lower Silesia: Wrocław, Leśnica [XS36] - Scholtz 1847, Scholz 1931, Assmann 1854. Pomeranian Lake District: Szczecin [VV71] - Schmidt 1928.

Western Beskidy Mts: Niedźwiedź [DV39] - Smreczyński 1906a, Smreczyński 1910. „Galicja”: Nowicki 1864.

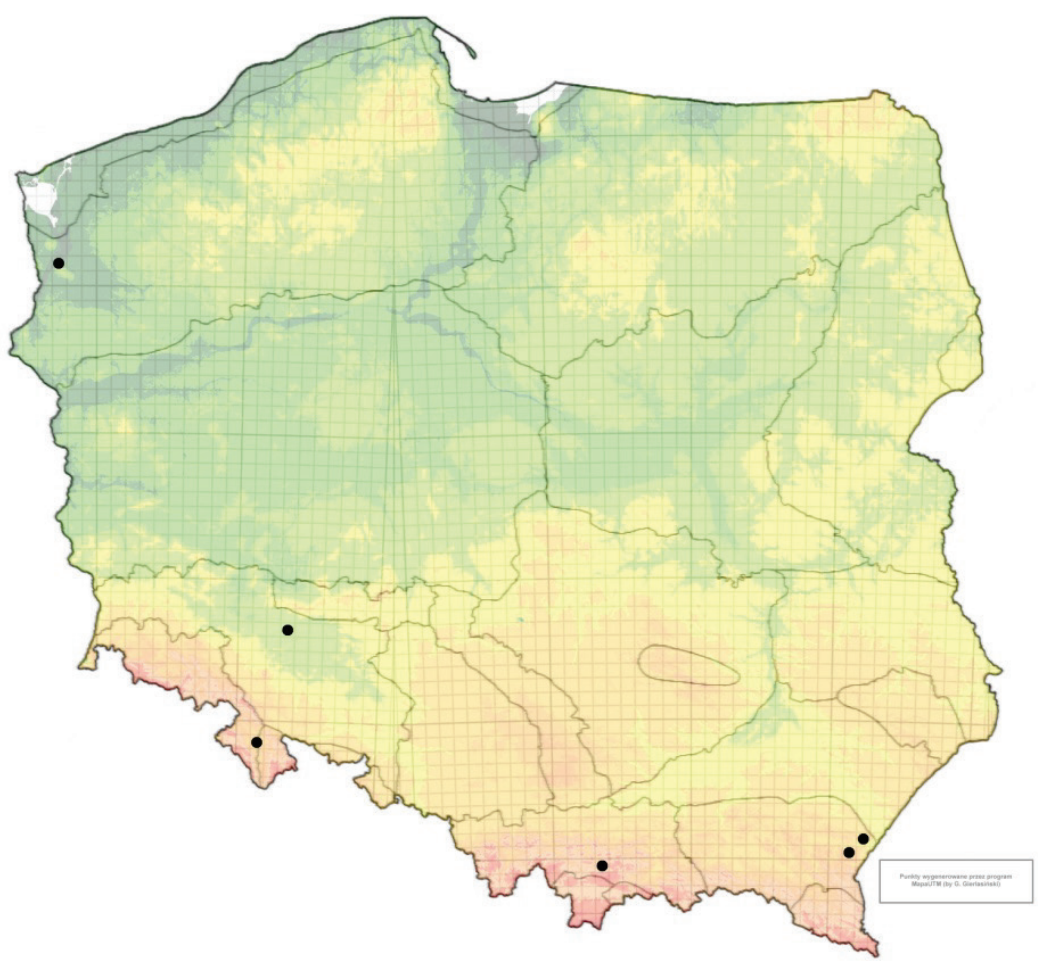

Fig. 9. Gampsocoris culicinus/punctipes - all data before 1948. 
Metacanthinae Douglas \& Scott, 1865

Metatropini Henry, 1997

\section{Metatropis rufescens (Herrich-Schäffer, 1835)}

Metatropis rufescens rufescens: Strawiński 1962: 184.

Metatropis lutescens: Strawiński 1964: 49.

Range element: Pacific-Atlantic.

Bionomics: Hygrophilous; skiophilous; oligophagous (Circaea spp., Linnaea borealis); one generation a year.

Remarks: Formerly considered a rare species in Poland (Hebda 1998, Gorczyca 2004), however in recent years, this species is regularly recorded in our country, as well as throughout Central Europe (Špryňar \& Kment, Taszakowski \& Gorczyca 2018). In Orzechowski \& Wasielewski 2016 the UTM square was incorrectly specified (correct is WT28).

Distribution in Poland (Fig. 10):

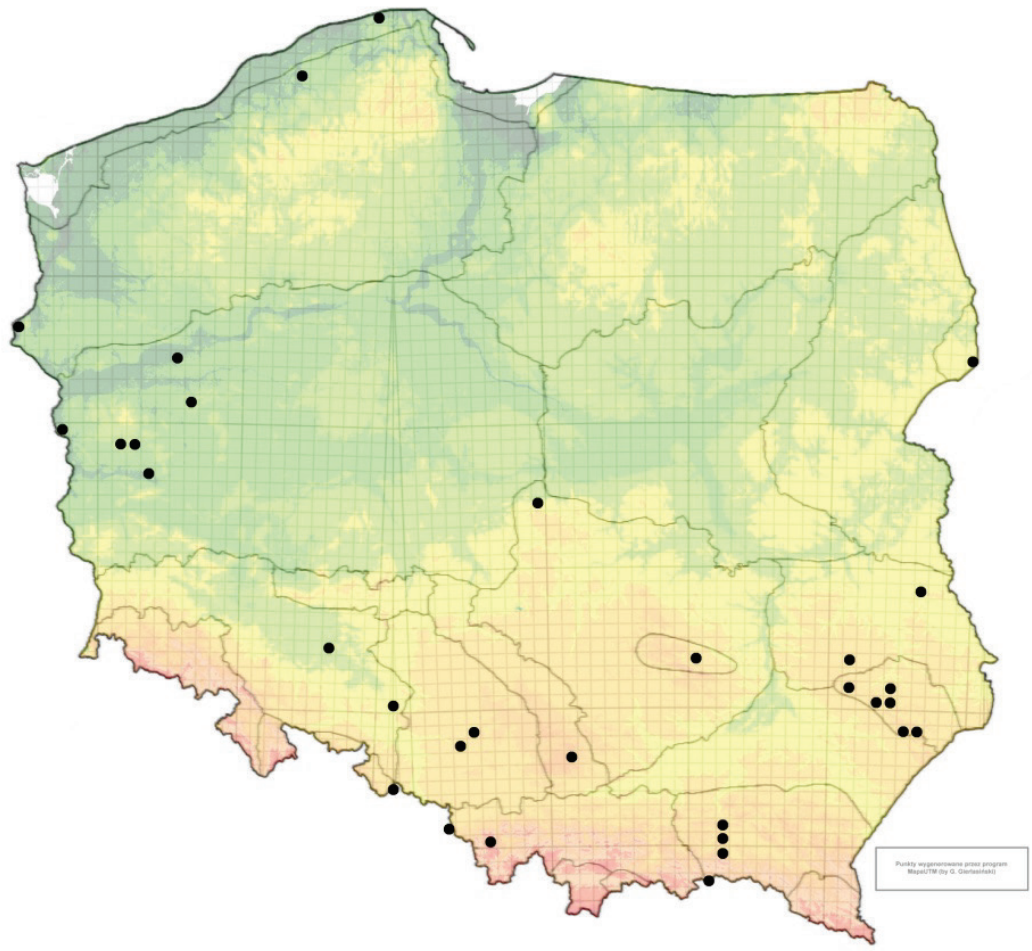

Fig. 10. Metatropis rufescens - distribution in Poland.

Baltic Coast: *Szklana Huta [XA87] - 1 ex., 26 Aug 2008, leg. M. W. Kozłowski.

Białowieża Forest: Białowieski NP [FD94] - Strawiński 1956b, Hebda 2011; Białowieża [FD94] - Smreczyński 1954.

Eastern Beskidy Mts: Bednarka [EA20, EV29] - Taszakowski \& Gorczyca 2018; upper Ropa River val. [EV17] - Taszakowski 2012; Lisów [EA21] - Taszakowski \& Gorczyca 2018. 
Eastern Sudetes Mts: Rozumice [YR14] - Hebda \& Kocorek 2012.

Krakowsko-Wieluńska Upland: Chełmowa Mt. [DA16] - Chłond \& Gorczyca 2009.

Lower Silesia: general - Lancke \& Polentz 1942; Górażdże [BB80] - Hohol-Kilinkiewicz \& Czaja 2006; *Oława [XS64] - 22 May 2014, 1 ex., leg. W.T. Szczepański (DZUS).

Lubelska Upland: Bachus Res. [FB68] - Cmoluchowa \& Lechowski 1990, Dziedzic \& Łętowski 2002; Sawin [FB68] - Cmoluchowa \& Lechowski 1992.

Małopolska Upland: *Łódź, Łagiewniki forest [CC94] - 23 May 1997, 1 ex., leg.

R. Dobosz (USMB).

Pomeranian Lake District: Bielinek over Odra River Res. [VU46] - Lis B. 2010; Słupsk [XA33] - Karl 1935.

Roztocze Upland: Jarugi Res. [FB41] - Cmoluchowa \& Lechowski 1994; Frampol [FB11] - Lechowski \& Smardzewska-Gruszczak 1996; Nart Res. [FB40], Stokowa Mt. [FB40], Tarnawa [FB13] - Cmoluchowa \& Lechowski 1994; Paary [FA68] - Strawiński 1959c; Bukowa Góra Res. [FB30] - Cmoluchowa \& Lechowski 1994, Strawiński 1964; Susiec [FA58] - Strawiński 1959a.

Świętokrzyskie Mts: Łysa Góra Mt. [EB03] - Strawiński 1962.

Upper Silesia: Segiecki forest [CA48] - Hebda 1998; *Segiet Res. [CA48] - 11 May 2009, 2 exx., 23 May 2010, 1 ex., leg. R. Dobosz (USMB), 11 Jul 2002, 7 exx., 1 Aug 2002, 5 exx., 21 Aug 2002, 1 ex., 1 Sep 2002, 1 ex., 11 Sep 2002, 1 ex., leg. A. Nosol (DBUO); *Zabrze [CA37] - 12 Jul 2012, 1 ex., leg. M. Obrzut (DZUS).

Western Beskidy Mts: *Cieszyn [CA21] - 22 Aug 2006, 1 ex., leg. J. Spandel (DBUO); Stary Groń Mts. [CA50] - Matuszczyk \& Taszakowski 2017.

Wielkopolsko-Kujawska Lowland: *Gościm, Solecko lake [WU54] - 1 ex, 3 Jun 2014, leg. R. Orzechowski; *Gryżyna, ad Gryżyńskie lake [WT18] - 1 ex., 2-4 Jun 2016, leg. M. Bunalski; *Krępa [WT36] - 1 ex., 16 Sep 2016, leg. R. Orzechowski; *Łęgi Słubickie [VT79] - 1 ex., 6 Sep 2016, 1 ex., 24 Oct 2016, leg. M. Adamski; *Mniszki [WU61] - 1 ex., 8 Sep 2016, leg. R. Orzechowski; Węgrzynice [WT28] - Orzechowski \& Wasielewski 2016.

„Pomerania”: Stichel 1926.

\section{DISCUSSION}

Currently, the occurrence of nine species of Berytidae (Fig. 11) is well documented in the Polish fauna, but we are aware that this number can change in future, mainly due to the detection of species previously unrecorded from Poland. The faunistic data from neighbouring countries (Péricart 2001) suggest that the following species can also be found in Poland:

- Berytinus (Lizinus) consimilis (Horváth, 1885) - known from the Czech Republic and Slovakia;

- Berytinus (Lizinus) distinguendus (Ferrari, 1874) - known from the Czech Republic and Ukraine;

- Berytinus (Lizinus) geniculatus (Horváth, 1885) - known from theCzech Republic, Germany, Slovakia and Ukraine;

- Berytinus (Lizinus) montivagus (Meyer-Dür, 1841) - the Czech Republic, Germany, Slovakia and Ukraine.

- Berytinus (Lizinus) striola (Ferrari, 1874) - known from the Czech Republic, Slovakia and Ukraine;

- Metacanthus (Cardopostethus) annulosus (Fieber, 1859) - reported form Slovakia, the Czech Republic (Vašíček et al. 2018) and Ukraine. 

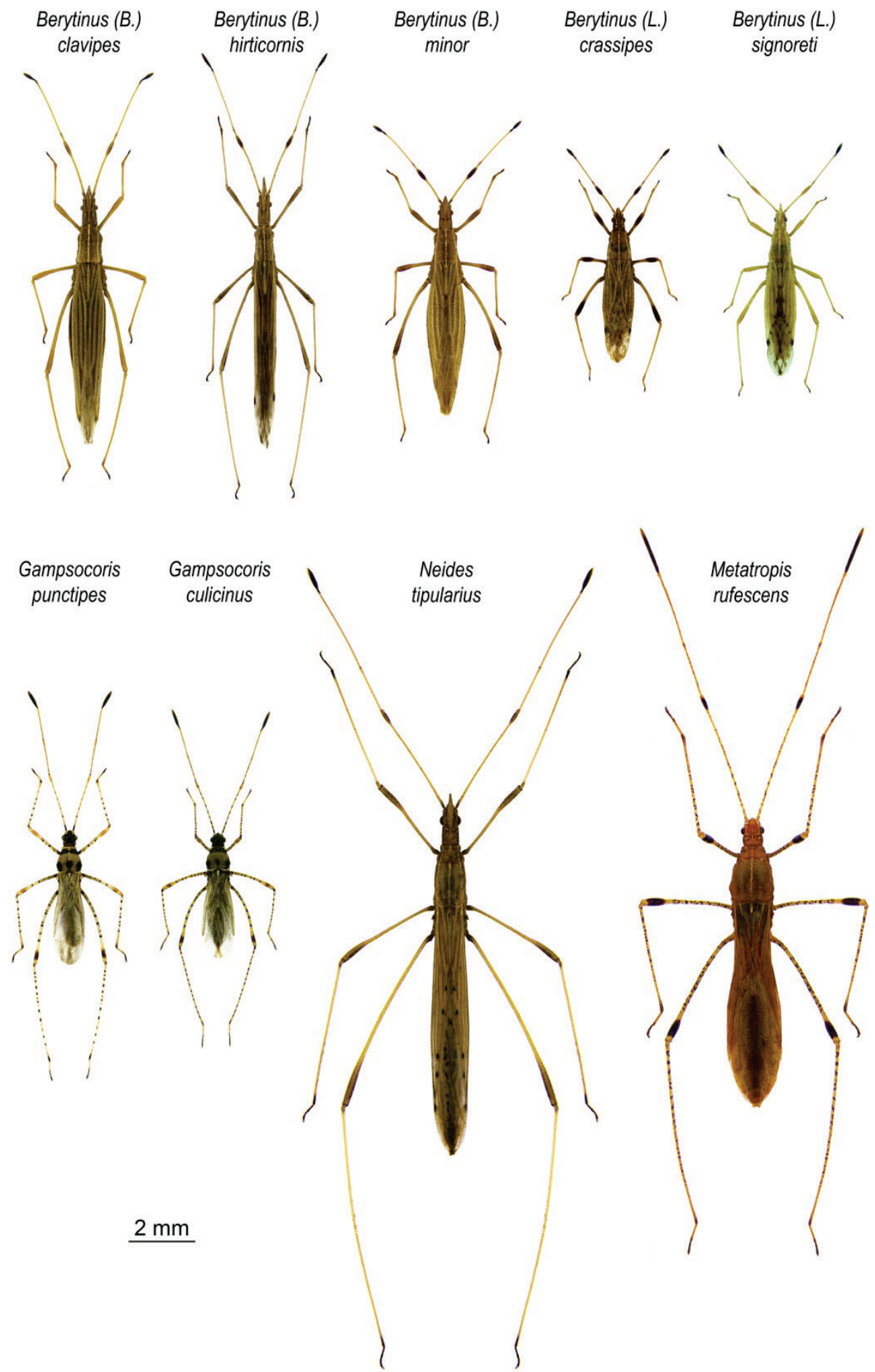

Fig. 11. All representatives of Berytidae occuring in Poland. Photos by A. Taszakowski. 
Additionally, we suggest to exclude Berytinus (Lizinus) montivagus (Meyer-Dür, 1841) from the Polish fauna. This species was listed from our country in several very old papers (Kotula 1890, Smreczyński 1906b, Schmidt 1928, Scholz 1931). The two first reports on this species (Kotula 1890, Smreczyński 1906b) were proved to be a case of misidentification of specimens represented B. signoreti and B. minor, respectively (Smreczyński 1954). Two subsequent reports, from Święta [VV73] in the Pomeranian Lake District (Schmidt 1928:195) and from Pątnów Legnicki [WS67] in Lower Silesia (Scholz 1931:155) are impossible to verify at present. All these facts show that there are no reliable reports of this species in Poland.

Moreover, in the "Catalogue of the Heteroptera of the Palaearctic Region" (Aukema \& Rieger 2001), B. montivagus was not listed from Poland, whereas Péricart (1984) placed a question mark next to this record from Poland with a note "probably absent". For the above reasons, exclusion of $B$. montivagus from the Polish fauna is justified for now; on the other hand, its occurrence in our country is quite possible because it was reported from Germany, the Czech Republic, Slovakia and Ukraine.

\section{ACKNOWLEDGEMENTS}

We are greatly indebted to M. Bunalski, J. Kalisiak, M. W. Kozłowski, J. Regner, M. Adamski, R. Matuszczak for sharing the data, T. Huflejt (ZMPA) and R. Dobosz (USMB) for the loan of the material for our study and to Petr Kment, whose tips greatly improved the current version of the manuscript. Our special thanks go to Dr Andrzej Palaczyk, for his kind assistance in verification of the identification correctness of the Berytinus hirticornis specimens deposited in the Museum of Natural History, Institute of Systematics and Evolution of Animals PAS, Kraków.

\section{REFERENCES}

AssmanN A. 1854. Hemiptera. Verzeichnis der bisher in Schleesien aufgefundanen wanzenartigen Insekten. Zeitschrift für Entomologie 8: 1-106.

Aukema B., Rieger CH. (ed.) 2001. Catalogue of the Heteroptera of the Palaearctic Region. Volume 4. The Netherlands Entomological Society, Wageningen: XIV + 346 pp.

BiLEWICZ-PAwiŃSKA T. 1961. The influence of wooded land on numerical dynamics of Heteroptera. Ekologia Polska A, 9: 61-77. [In Polish with English Summary]

BILEWICZ-PAwiŃSKA T. 1965. Ecological analysis of Heteroptera communities in cultivated fields. Ekologia Polska 13: 593-639.

Bugaj-NAwrocka A. \& Gorczyca J. 2013. The terrestrial true bugs (Hemiptera: Heteroptera) of Kocie Górki, Piekary Śląskie (Upper Silesia). Wiadomości entomologiczne 32 (2): 118-126. [In Polish with English Abstract and Summary]

BugAj-NAWrockA A., WieczoreK K. \& HerczeK A. 2018. Terrestrial true bugs (Hemiptera: Heteroptera) communities of selected phytocoenoses of the Trzebnica Hills. Monographs of the Upper Silesian Museum 9: 1-97.

Burakowski B., Mroczkowski M. \& StefańsKa J. 1973. Chrząszcze Coleoptera. Biegaczowate - Carabidae. Część 1. Katalog Fauny Polski 23 (2): 1-232.

BuRDAJEWICZ S. \& NowACKA W. 1995. Entomofauna of the glades in the forest district of Gwda Wielka. Part II. Bugs (Heteroptera). Entomological News 14 (1): 21-25. [In Polish with English Abstract]

CELARY W. 2003. The invertebrate fauna (Invertebrata) of Mt. Babia Góra, not included in separate chapters. Monografia Fauny Babiej Góry, 373-398. [In Polish with English Abstract and Summary]

ChŁond D., ĆWIKŁa J. \& GRomotKa P. 2005. Heteropteran terrestrial bugs of the „Dolina Żabnika” nature reserve (Hemiptera: Heteroptera). Acta Entomologica Silesiana 12: 19-27. [In Polish with English Abstract]

CHŁOND D. \& GoRCZYCA J. 2009. Terrestrial true bugs (Hemiptera, Heteroptera) of the Ojców National Park - Origin of Fauna. Annals of the Upper Silesian Museum, Entomology 17: 5-109.

CMoluch Z. 1960. Entomofauna rzepaku ozimego - Brassica napus L. var. biennis (Schubler \& Mart.). Polskie Pismo Entomologiczne 19: 167-182.

Cmoluchowa A. 1958. Hemiptera-Heteroptera łąk okolic Zemborzyc i Wrotkowa. Annales Universitatis Mariae Curie-Skłodowska, C, 13: 165-189. 
Cmoluchowa A. 1960. Observations on Hemiptera-Heteroptera of the botanical garden of the Maria CurieSkłodowska University, Lublin. Annales Universitatis Mariae Curie-Skłodowska, C, 15: 161-175.

CMoluchowa A. 1964. Pluskwiaki różnoskrzydłe (Hemiptera-Heteroptera) roślinnych zespołów kserotermicznych okolic Kazimierza nad Wisłą. Annales Universitatis Mariae Curie-Skłodowska, C, 19: 49-94.

Cmoluchowa A. 1971. Heteroptera of the dunes in the Lublin Region. Annales Universitatis Mariae CurieSkłodowska, C, Biologia 26 (12): 129-153.

CMOluchowa A. \& LeCHOWSKi L. 1977. Uzupełnienie do poznania pluskwiaków rożnoskrzydłych (Heteroptera) Beskidu Wschodniego i Bieszczadów. Annales Universitatis Mariae Curie-Skłodowska, C, 21: 265-269.

CMoluchowa A. \& LeCHOWSKi L. 1988. Species composition and numerical force of Heteroptera of the Lublin Coal Basin. Annales Universitatis Mariae Curie-Skłodowska, C, 40: 75-84.

Cmoluchowa A. \& LechowSKi L. 1990. Pluskwiaki różnoskrzydłe (Heteroptera) zespołu grądowego (TilioCarpinetum) w rezerwacie Bachus (Wyżyna Lubelska). Fragmenta Faunistica, 33: 361-371. [In Polish with English Abstract and Summary]

CMOluChowA A., LeChOwSKi L. 1992. Changes in Heteroptera groups in dry-ground forest communities ad Sawin, Chełm Province. Annales Universitatis Mariae Curie-Skłodowska, C, 44: 79-90.

Cmoluchowa A. \& LechowsKi L. 1993. Heteroptera communities of pine forests in Poland. Fragmenta Faunistica 36(8): 127-146.

CMOluchowa A. \& Lechowski L. 1994. Lądowe pluskwiaki różnoskrzydłe (Heteroptera) Roztocza. Fragmenta Faunistica 37 (7): 181-199. [In Polish with English Abstract and Summary]

DOHRN A. 1860. Hemipterologische Miscellaneen II. Stettiner Entomologische Zeitung 21: 99-109, $158-161$.

DomagalA P. \& GRADOWSKA P. 2017. Metatropis rufescens (Herrich-Schäffer, 1835) (Heteroptera: Berytidae): the second record in the Eastern Sudetes, and the first in the Góry Opawskie Mountains. Acta Entomologica Silesiana 25: 38.

DZIEDZIC R. \& ŁęTOWSKI D. 2002. Zoo-cenoses of the land (forest, meadow and agrocultural) ecosysytems of the Lublin Polesie - current state and direction of changes. Acta Agrophysica 66: 179-195. [In Polish with English Summary]

ENGEL H. 1938. Beitrage zur Flora und Fauna der Binnendune bei Bellinchen (Oder). Markische Tierwelt 3: $229-294$.

Engel H. \& Hedicke H. 1934. Die Fauna der Binnendune bei Bellinchen (Oder). IV. Die Tierwelt. Heteroptera. Markische Tierwelt 17: 240-246.

FEDORKO J. 1957. Wstępne badania nad heteropterofauną ściółki leśnej na przykładzie materiału z Wandzina. Annales Universitatis Mariae Curie-Skłodowska, C, 12: 205-237.

FEDORKO J. 1959. An attempt to find the relationship between the occurence of Heteroptera and forest habitats. Annales Universitatis Mariae Curie-Skłodowska, C, 14: 93-115.

GorczYCA J. \& LIS J. A. 2000. Terrestrial true bugs (Heteroptera) in Bieszczady Mountains. Monografie Bieszczadzkie 7: 191-204. [In Polish with English Abstract and Summary]

GORCZYCA J. 2004. Lądowe pluskwiaki różnoskrzydłe Heteroptera. In: BogDANOwicz W., CHUdZICKA E., PILIPIUK I. \& SKIBIŃSKA E. (eds), Fauna of Poland - Characteristics and checklist of species. Vol I. Annelida, Arthropoda pro parte, Insecta pro parte (Coleoptera, Hemiptera, Hymenoptera, Lepidoptera). Pp. 192-203, 213-234. Muzeum i Instytut Zoologii PAN. Warszawa 509 pp. [In Polish with English summaries]

GRAVENHORTS I. L. C. 1836. Jahres-Bericht uber die Arbeiten der entomologischen Section. Ubersicht der Arbeiten und Veranderungen Schlesischen Gesellschaft fur Vaterlandische Kultur 1835: 79-83.

GruHL K. 1929. Tier und Pflanzenwelt des Kreises Grunberg in Schlesien und seiner naheren Umbegund. Levysohn, Grunberg. 587 pp.

HAŁKA-WoJCIECHOWICZ E. 1996. Zgrupowania pluskwiaków różnoskrzydłych (Heteroptera) zasiedlające trawniki miejskie wybranych osiedli mieszkaniowych Warszawy. Fragmenta Faunistica, 39 (10): 127-148. [In Polish with English Abstract]

HEBDA G. 1998. First record of Metatropis rufescens (Herrisch-Schaffer, 1835) (Insecta: Heteroptera) in Upper Silesia. Natura Silesiae Superioris 2: 81-84. [In Polish with English Abstract and Summary]

HeBDA G. 2006. Heteropterans (Insecta: Heteroptera) on limestone quarries in the Chelm area (Opole Silesia). In: Nowak A., Hebda G. (eds.), Biodiversity of quarries and pits. Opole Scientific Society, 3rd Department of Natural Sciences, Opole-Górażdże: 83-94.

HebDa G. 2011. New data on the distribution of terrestrial true-bugs (Hemiptera: Heteroptera) in the Białowieża Primeval Forest. Heteroptera Poloniae - Acta Faunistica, 3: 11-19. [In Polish with English Abstract]

HeBdA G. \& KocoReK A. 2012. Pluskwiaki różnoskrzydłe (Hemiptera: Heteroptera) leśnego rezerwatu przyrody Rozumice na Opolszczyźnie (Sudety Wschodnie). Heteroptera Poloniae. Acta Faunistica 5: 9-16. [In Polish with English Abstract]

HEBDA G. \& RUTKOWSKI T. 2015. First data on the occurrence of terrestrial true-bugs (Hemiptera: Heteroptera) in Ujście Warty National Park. Wiadomości Entomologiczne 34 (1): 12-18. [In Polish with English Abstract and Summary]

HeBDA G. \& ŚCIBIOR R. 2013. Gampsocoris culicinus Seidenstucker, 1948, species new to the Polish fauna (Heteroptera: Berytidae: Gampsocorinae). Genus 24: 29-32. 
Hebda G. \& ŚciBior R. 2016. New localities of terrestrial bugs (Hemiptera: Heteroptera) in Pieniny Mts.. Parki Narodowe i Rezerwaty Przyrody 35 (4): 93-98. [In Polish with English Abstract and Summary]

Henry T. J. 2000. Stilt Bugs (Berytidae). In: Schaefer C. W. \& PANizZi A. R. (eds.), Heteroptera of Economic Importance. Environment and Agriculture, CRC Press, $856 \mathrm{pp}$.

HenRY T. J. \& FroeschneR R. C. 1998. Catalog of the stilt bugs, or Berytidae, of the world (Insecta: Hemiptera: Heteroptera). Contributions of the American Entomological Insititute 30 (4): 1-72.

Hohol-KilinKiewicz A. \& CZAJA J. 2006. Heteroptera (Insecta: Hemiptera) of Górażdże quarry (Lower Silesia) preliminary study. In: NowAK A. \& HEBDA G. (eds), Biodiversity of quarries and pits. Opole Scientific Society, 3rd Department of Natural Sciences, Opole-Górażdże: 137-144.

KARL O. 1935. Ein Beitrag zur Hemipterenfauna Ostpommerns. Wanzen, Zikaden und Blattflöche. Dohrniana, Abhandlungen und Berichte der Pommerschen Naturforschenden Gesellschaft 14: 122-141.

KĘDZIOR M., LIS J.A. \& WOLSKI A. 2012. Pluskwiaki różnoskrzydłe (Hemiptera: Heteroptera) masywu Czantorii Wielkiej (Beskid Śląski). Heteroptera Poloniae - Acta Faunistica, 5: 17-24. [In Polish with English Abstract]

KONDRACKI J. 2011. Geografia regionalna Polski. Wydawnictwo Naukowe PWN. Warszawa: 440 pp.

KoRCZ A. 2003. Heteroptera in diverse natural habitats of Słowiński National Park and the Rowokół Hill. Rozprawy Naukowe Instytutu Ochrony Roślin 13: 201 pp. [In Polish with English Summary]

Kosicki S. 1958. Lądowe pluskwiaki różnoskrzydłe (Hemiptera, Heteroptera) rezerwatu cisowego Wierzchlas i terenów przyległych. Zeszyty naukowe Uniwersytetu Mikołaja Kopernika w Toruniu. Biologia 2: 117-144.

Kotula B. 1890. Spis pluskiew z okolic Przemyśla (i po części Lwowa). Sprawozdanie Komisji Fizyograficznej PAU 25: $131-140$.

KRASUCKI A. 1919. Przyczynek do poznania fauny pluskwiaków krajowych (Sprawozdanie z wycieczki do Zarzecza, odbytej w sierpniu w r. 1917). Rozprawy i wiadomości z muzeum im. Dzieduszyckich 3: 191-198.

Kuhlgatz T. 1901. Orthoptera, Odonata und Rhynchota. In: RüBSAAMEN E. H. (eds) Bericht über meine Reisen durch die Tucheler Heide in den Jahren 1896 und 1897. Danzig. 145-148.

LANZKE A. \& Polentz G. 1942. Beitrage zur Kenntnis der schlesischen Wanzen. Zeitschrift fur Entomologie, 19: 11-14.

LECHOWSKI L. 1984. Studies on Heteroptera fauna in plant communities of the Bystrzyca Valley. I. Phytophagous insect Fauna. Annales Universitatis Mariae Curie-Skłodowska, C, 39 (28); 219-241. [In Polish with English and Russian Summary]

LECHOWSKI L. 1989. Heteroptera of moist meadows on the Mazovian Lowland. Memorabilia Zoologica, 43: 119-126.

Lechowski L. \& CMoluchowa A. 1993. The Qualitative and Quantitative Structure of the Fauna of the Order Heteroptera in the Bukowa Gora Reservation (Roztocze National Park). Annales Universitatis Mariae CurieSkłodowska, C, 51 (8): 51-57. [In Polish with English Summary]

Lechowski L. \& SMARdZEWSKA-GruszczaK Z. 1996. Preliminary studies on Heteroptera Fauna of the Landscape Park Lasy Janowskie. In: RadWAn S., SAŁATA B. \& Szunke Z. Walory przyrodnicze Parku Krajobrazowego Lasy Janowskie, pp. 73-74. Wydawnictwa UMCS, Lublin. 84 pp.

LECHOWSKI L. \& SMARDZEWSKA-GRUSZCZAK Z. 1998. Bugs (Heteroptera) of the Szklarnia reserve („Lasy Janowskie" Landscape Park). Parki Narodowe i Rezerwaty Przyrody 17: 57-65. [In Polish with English Abstract]

LECHOWSKI L. \& SMARDZEWSKA-GRUSZCZAK Z. 2004. Heteroptera of the peat-bog reserve Bagno Serebryskie (Serebryskie Swamp) near Chełm. Annales Universitatis Mariae Curie-Skłodowska, C, 59: 43-50.

LECHOWSKI L. \& SMARDZEWSKA-GRUSZCZAK Z. 2006a. Land Heteroptera of some habitats of Polesie Wołyńskie. Wiadomości entomologiczne, 25 (Supl. 2): 131-134. [In Polish with English Abstract and Summary]

LECHOWSKI L. \& SMARDZEWSKA-GRUSZCZAK Z. 2006b. Lądowe Heteroptera wybranych siedlisk Polesia Wołyńskiego. IV Ogólnopolska Konferencja Naukowa, Zwierzyniec, Materiały Konferencyjne.

LIS B. 1994. Pluskwiaki różnoskrzydłe (Heteroptera) Kamiennej Góry w Ligocie Dolnej (Górny Śląsk). Acta Entomologica Silesiana 2: 25-30. [In Polish with English Abstract]

Lis B. 2007. Płaszczyńcowate - Piesmatidae, smukleńcowate - Berytidae, kowalowate - Pyrrhocoridae. Klucze do oznaczania owadów Polski 18 (9): 33 pp.

Lis B. 2010. Pluskwiaki różnoskrzydłe (Hemiptera: Heteroptera) rezerwatu leśno-stepowego Bielinek nad Odrą $\mathrm{i}$ jego okolic (Pojezierze Pomorskie). Heteroptera Poloniae - Acta Faunistica 2: 37-49. [In Polish with English Abstract]

Lis B., MĄsior J. \& Lis J. A. 2002. True-bugs (Hemiptera: Heteroptera) of the Babia Góra Mountain (West Beskid Mts.). Wiadomości Entomologiczne 20: 103-111. [In Polish with English Abstract and Summary]

Lis B., WolSKI A. \& LIS J.A. 2004. Charagochilus weberi Wagner, 1953 (Hemiptera: Heteroptera: Miridae) - a species new to the Polish fauna, and a list of species collected in the Polish Tatras. Polskie Pismo Entomologiczne, 73: $317-322$.

Lis B. \& DANiElczoK-Demska T. 2001. Pluskwiaki różnoskrzydłe (Insecta: Heteroptera) Parku Krajobrazowego Góra św. Anny (Górny Śląsk). Natura Silesiae Superioris, 5: 53-59. [In Polish with English Abstract and Summary]

Lis B. \& DuBIEL G. 2013. Acetropis longirostris Put. and Oxycarenus pallens (H.-S.) - two species of true-bugs (Hemiptera: Heteroptera) new for the Polish fauna, with a checklist of species collected in a vicinity of Bystra in the Silesian Beskid. Heteroptera Poloniae - Acta Faunistica 7: 33-44. [In Polish with English Abstract] 
Lis B. \& KowAlCZYK J. K. 2017. Terrestrial true-bugs (Hemipter: Hetroptera) of Gdynia (Polish Baltic Coast). Heteroptera Poloniae - Acta Faunistica 11: 51-63. [In Polish with English Abstract and Summary]

Lis J. A. 1989. True-bugs (Insecta: Heteroptera) of the Silesian Upland. Annals of the Upper Silesian Museum in Bytom, Natural History 12: 5-60. [In Polish with English Abstract and Summary]

Lis J. A., Lis B. \& GoRCZYCA J. 1995. Bugs (Heteroptera) of the middle basin of the Biebrza River Valley. Entomological news 14 (2): 85-93. [In Polish with English Abstract]

Lis J. A. \& LIS B. 1998. True-bugs of Śląsk Górny - an annotated checklist (Hemiptera: Heteroptera). Roczniki Muzeum Górnośląskiego w Bytomiu, Entomologia 8: 107-146.

ŁĘGOWSKI D. \& LIS B. 2008. New data on terrestrial bugs (Hemiptera: Heteroptera) of the Hel Peninsula (Baltic Coast). Opole Scientific Society Nature Journal, 41: 117-131. [In Polish with English Abstract and Summary]

ŁOMNICKI M. 1882. Pluskwy różnoskrzydłe (Hemiptera-Heteroptera) znane dotychczas z Galicyi. Sprawozdanie Komisji Fizyograficznej PAU 16: 37-55.

MATUSZCZYK J. \& TASZAKOWSKi A. 2017. Terrestrial true-bugs (Hemiptera: Heteroptera) of the Stary Groń massif (Silesian Beskid Mts.). Heteroptera Poloniae - Acta Faunistica 11: 65-71. [In Polish with English Abstract and Summary]

MAZUR S. 2001. Ryjkowce kserotermiczne Polski: (Coleoptera: Nemonichidae, Attelabidae, Apionidae, Curculionidae). Studium zoogeograficzne. Monografie Fauny Polski 22: 378 pp. [In Polish with English Summary]

MiKOŁAJSKI M. 1961. Z badań nad występowaniem pluskwiaków różnoskrzydłych (Hemiptera-Heteroptera) na uprawach lucerny w województwie olsztyńskim. Zeszyty Naukowe Wyższej Szkoły Rolniczej w Olsztynie 11: $245-267$.

MiKOŁAJSKI M. 1962a. Observations upon Hemiptera-Heteroptera in peatbog-reservation „Redykajny” in Neighbourhood Olsztyn. Studia Societatis Scientiarum Torunensis Sectio E (Zoologia), 6: 211-227.

MiKOŁAJSKI M. 1962b. Obserwacje nad pluskwiakami różnoskrzydłymi (Hemiptera-Heteroptera) upraw koniczyny czerwonej (Trifolium pratense L.) w województwie olsztyńskim. Zeszyty Naukowe Wyższej Szkoły Rolniczej w Olsztynie 14: 119-126. [In Polish with Russian Summary]

MiKOŁAJSKI M. 1962c. Tytthus pygmaeus (Zett.) - a new species to the fauna of Poland and some other species of Hemiptera-Heteroptera new to the fauna of the Warmia-Mazury Region. Fragmenta faunistica, 10 (17): $251-255$. [In Polish with English Summary]

MiKOŁAJSKI M. 1962d. Z badań nad heteropterofauną łąk doliny rzeki Łyny pod Olsztynem. Zeszyty Naukowe Wyższej Szkoły Rolniczej w Olsztynie 14: 105-118. [In Polish with English Summary]

MORKEL C. 2007. On kleptoparasitic stilt bugs (Insecta, Heteroptera: Berytidae) in spider funnel-webs (Arachnida, Araneae: Agelenidae), with notes on their origin. The Science of Nature 31:129-143.

MusiK K. 2010. Terrestial true bugs (Hemiptera: Heteroptera) of „Żabie Doły” natural-landscape area near Bytom.. Acta Entomologica Silesiana, 18: 23-29. [In Polish with English Abstract]

NoGA P. \& LIS B. 2015. Results of faunistic studies on true-bugs (Hemiptera: Heteroptera) of selected meadow phytocoenoses of Przedmość (Opole voivodeship). Heteroptera Poloniae - Acta Faunistica 9: 51-79. [In Polish with English Abstract and Summary]

NOwICKI M. 1864. Przyczynek do owadniczej fauny Galicyi. Rozprawy i Wiadomości Muzeum Dzieduszyckich, Kraków, 87 pp.

NowICKI M. 1868. Wykaz pluskwówek (Rhynchota F., Hemiptera). Sprawozdanie Komisji Fizyograficznej PAU 2: 91-107.

NowICKI M. 1870. Dodatek do wykazu pluskwiaków. Sprawozdanie Komisji Fizyograficznej PAU 4: 237-240.

ORZECHOWSKi R. \& WASIELEWSKi H. 2016. Owady Gryżyńskiego Parku Krajobrazowego - pozostałe grupy. In: MACiANTOWiCZ M. (ed.), 20 lat - Gryżyński Park Krajobrazowy - Monografia przyrodnicza:.180-195. Zespół Parków Krajobrazowych Województwa Lubuskiego, Gorzów Wielkopolski [In Polish with English Summary]

PiASECKA J. 1960. Hemiptera - Heteroptera found on the Meadows of Janów Lubelski Situated in the Close Vicinity of Forests. Annales Universitatis Mariae Curie-Skłodowska, C, 15 (4): 89-109. [In Polish with English Summary]

PÉRICART J. 1984. Hémiptères Berytidae euro-méditerranéens. Faune de France, 70. Fédération Française des Sociétés de Sciences Naturelles, Paris, $171 \mathrm{pp}$.

PÉRICART J. 2001. Family Berytidae Fieber, 1851 - Stilt-bugs. In: Aukema B., RiEger CH. (eds), Catalogue of the Heteroptera of the Palaearctic Region. Volume 4. The Netherlands Entomological Society, Wageningen: 230-242.

PolentZ G. 1943. Beitrage zur Kenntnis der schlesischen Wanzen. Zeitschrift für Entomologie 19 (2): 9-14.

SCHMIDT E. 1928. Verzeichnis der pommerschen Wanzen nach dem Material des Pommerschen Museum für Naturkunde. Abhandlungen und Berichte der Pommerschen Naturforschenden Gesellschaft 9: 188-196.

Scholtz H. 1847. Prodromus zu einer Rhynchoten. Fauna von Schlesien. Übersicht der Arbeiten und Veranderungen Schlesischen Gesellschaft für Vaterlandische Kultur 1846: 104-164.

Scholz M. F. R. 1931. Verzeichnis der Wanzen Schlesiens. Entomologischer Anzeiger. Spezialzeitschrift für entomologischen Handel, Verkehr und Literatur, Wien 11: 78-92, 99-102, 117-120.

Schumacher F. 1912. Die Rhynchoten-Fauna der Mark Brandenburg IV. Fam. Berytidae (Neididae). Berliner Entomologische Zeitschrift 57: 131-143. 
SCHUMACHER F. 1913. Weitere Beitrage zur Hemipteren-Fauna der Provinz Posen. Deutschen Entomologischen Gesellschaft 6: 670-674.

SCHUMMEL T. E. 1836. Seltene Hemipteren aus Schlesien. Ubersicht der Arbeiten und Veranderungen Schlesischen Gesellschaft für Vaterlandische Kultur 1835: 81.

SiEBold C. T. 1839. Beitrag zur Fauna der wirbellosen Tiere Preussens. IV Beitrag: Preussische Wanzen und Zirpen. Preussischen Provinzialblatter 21: 429-447.

SKóRKA S. 1994. Land-bugs (Heteroptera) of the Puszcza Zielonka forest near Poznań. Acta Entomologica Silesiana, $2(1): 13-20$.

SMARDZEWSKA-GRUSZCZAK Z. \& LECHOWSKi L. 2000. The Heteroptera of the planned peat-bog reserve Zawadówka. In: ŁĘTOWSKI J. (ed.), Walory przyrodnicze Chełmskiego Parku Krajobrazowego jego najbliższych okolic, pp. 123-134. Wydawnictwo UMCS, $215 \mathrm{pp}$.

SMARDZEWSKA-GRUSZCZAK Z. \& LeChOWSKI L. 2006. True bugs (Hemiptera: Heteroptera) of the Brzeźno nature reserve near Chełm. Annales Universitatis Mariae Curie-Skłodowska, C, 61: 89-97.

SMRECZYŃSKi S. 1906a. Wykaz pluskwiaków nowych dla fauny galicyjskiej. Sprawozdanie Komisji Fizyograficznej PAU 40: 72-79.

SMRECZYŃSKI S. 1906b. Zbiór pluskwiaków prof. Dra Stanisława Zaręcznego. Sprawozdanie Komisji Fizyograficznej PAU 40: 46-71.

SMRECZYŃSKi S. 1908. Dodatek do spisu pluskiew św. p. prof. B. Kotuli. Sprawozdanie Komisji Fizyograficznej PAU 43: $1-11$.

SMRECZYŃSKi S. 1910. Spis pluskwiaków zebranych w Gorcach w roku 1909. Sprawozdanie Komisji Fizyograficznej PAU 44: 109-122.

SMRECZYŃSKI S. 1954. Materiały do fauny pluskwiaków (Hemiptera) Polski. Fragmenta Faunistica 7: 1-146.

STICHEL W. 1926. Illustrierte Bestimmungstabellen der deutschen Wanzen (Hemiptera: Heteroptera). Lief. 4. Pp. 91120. Verlag naturwissenschaftlicher Publikationen, Berlin-Hermsdorf.

STEHLIK J.L. \& VAVŘínOVÁ I. 1990. Results of investigations on Heteroptera in Slovakia made by the Moravian Museum (Berytidae). Acta Musei Moraviae, Scientiae Naturales 75: 219-235.

StoBIECKi S. 1886. Materiały do fauny W. Ks. Krakowskiego. 1. Pluskwiaki (Hemiptera), Szarańczaki (Orthoptera) i Mięczaki (Mollusca). Sprawozdanie Komisji Fizyograficznej PAU 20: 120-161.

STOBIECKI S. 1915. Wykaz pluskwiaków (Rhynchota) zebranych w Galicji zachodniej i środkowej. Sprawozdanie Komisji Fizyograficznej PAU 49: 126-219.

STRAwIŃSKI K. 1936. Badania nad fauną pluskwiaków drzew i krzewów w Polsce. Rozprawy i sprawozdania Instytutu Badawczego Lasów Państwowych 17: 1-216.

STRAWIŃSKI K. 1953. Badania nad pluskwiakami (Heteroptera) żyjącymi na łąkach na przykładzie materiału z Iwonicza. Annales Universitatis Mariae Curie-Skłodowska, C, 8 (10): 357-401.

STRAWIŃSKI K. 1956a. Materials to the Hem.-Heteroptera of Roztocze. Annales Universitatis Mariae CurieSkłodowska, C, 11 (6): 151-181.

STRAwIŃSKi K. 1956b. Owady z rzędu Heteroptera w biocenozie Puszczy Białowieskiej. Roczniki Nauk Leśniczych 14: $3-101$.

STRAWIŃSKI K. 1956c. Badania nad ustaleniem składu jakościowego i ilościowego heteropterofauny żyta na polach śródleśnych i bezleśnych. Ekologia Polska 4: 95-169.

STRAWIŃSKI K. 1957a. Hemiptera-Heteroptera runa leśnego z okolic Wandzina. Annales Universitatis Mariae CurieSkłodowska, C, 12: 103-113.

STRAWIŃSKI K. 1957b. Hemiptera-Heteroptera w biocenozie łąk okolic Puław. Ekologia Polska, 5 (8): 111-125.

STRAWIŃSKI K. 1959a. Porównawcze badania nad Hemiptera-Heteroptera w kilku biotopach łąkowych w okolicach Suśca. Polskie Pismo Entomologiczne 29: 139-162.

STRAWIŃSKI K. 1959b. A Faunistic Survey of Hemiptera-Heteroptera Occuring in the „Steppe” Reservation near Gródek. Annales Universitatis Mariae Curie-Skłodowska, C, 14: 1-28.

STRAWIŃSKI K. 1959c. Heteroptera less known, more rare and new to the fauna of Poland. Fragmenta faunistica, 8: 133-136. [In Polish with English Summary]

STRAWIŃSKI K. 1960. Pluskwiaki różnoskrzydłe (Hemiptera-Heteroptera) śródleśnych środowisk z roślinnością kserotechniczną w okolicach Łabuń. Ekologia Polska, 5: 139-159. [In Polish with German Summary]

STRAWIŃSKI K. 1962. Hemiptera-Heteroptera in the Świętokrzyski National Park. Annales Universitatis Mariae CurieSkłodowska, C, 17: 165-193. [In Polish with Russian Summary]

STRAWIŃSKI K. 1963. Powiązania biocenotyczne owadów Hemiptera-Heteroptera z biotopami zadrzewionymi i niezadrzewionymi w okolicach Puław. Annales Universitatis Mariae Curie-Skłodowska, C, 18: 1-27.

STRAWIŃSKI K. 1964. Hemiptera-Heteroptera Found in the Forest Reserve Bukowa Góra near Zwierzyniec (Zamość District). Annales Universitatis Mariae Curie-Skłodowska, C, 19 (3): 37-48.

STRAWIŃSKI K. 1965. Zestawienie zgrupowań gatunków Hemiptera-Heteroptera niezacienionych biotopów śródleśnych w nadleśnictwie Duninów koło Płocka. Annales Universitatis Mariae Curie-Skłodowska, C, 20 (1): 1-8.

STRAWIŃSKI K. 1966a. Hemiptera-Heteroptera na nieużytkach śródpolnych w okolicy Zwierzyńca (powiat zamojski). Polskie Pismo Entomologiczne 43: 315-334. 
STRAWIŃSKI K. 1966b. The influence of some ecological habitat factors on Hemiptera-Heteroptera communities. Ekologia Polska 14: 215-225.

SZULCZEWSKI A. 1908. Verzeichnis der bei Janowitz im Kreise Znin gefangenen Wanzen. Zeitschrift der Naturwissenschaftliechen Abteilung 15: 35-38.

SzULCZEWSKI A. 1913. Beitrag zu einem Verzeichnis der Posener Rhynchoten (Hemiptera). Deutschen Entomologischen Gesellschaft 3: 307-314.

TARNAWSKI D. 2013. True-bugs (Hemiptera: Heteroptera) of Zakrzewska Osada (Krajna) in Pomeranian Lakeland. Heteroptera Poloniae - Acta Faunistica 7: 9-32. [In Polish with English Abstract]

TASZAKOWSKI A. 2012. Terrestrial true bugs (Hemiptera: Heteroptera) of the upper Ropa river valley. Acta entomologica silesiana 20: 37-54. [In Polish with English Abstract]

TASZAKOWSKI A. 2015. Notes on the occurrence of Gampsocoris Fuss, 1852 (Hemiptera: Heteroptera: Berytidae) in Poland. Fragmenta Faunistica, 58 (1): 1-6.

TASZAKOWSKi A. \& PASIŃSKA A. 2017. New data on the occurence of terrestrial true-bugs (Hemiptera: Heteroptera) in Pieniny Mountains. Fragmenta Faunistica 60 (1): 1-13.

TASZAKOWSKI A. \& GORCZYCA J. 2018. Terrestrial true-bugs (Hemiptera: Heteroptera) of the Eastern Beskidy Mountains - origin of fauna. Monograph of the Upper Silesian Museum in Bytom 8: 1-159.

TenenBAum S. 1921. Pluskwiaki (Rhynchota) z Ordynacji Zamojskiej. Pamiętnik Fizjograficzny 26: 1-16.

TOMASIK Ł. 2014. Inwentaryzacja i waloryzacja przyrodnicza doliny rzeki Pacynki wraz z doliną cieku Mnich w granicach administracyjnych Radomia (pow. 220 ha). Radom. 138 pp.

TrOJAN P. 1989. Bug (Heteroptera) associations in the agricultural landscape of Great Poland. Ekologia Polska 37: 135-155.

Trojan P., Bańkowska R., Chudzicka E., Pilipiuk I., Skibińska E., Sterzyńska M. \& Wytwer J. 1994. Secondary succesion of fauna in the pine forests of Puszcza Białowieska. Fragmenta Faunistica 37: 3-104.

VAŠ́́ČEK M., CUNEV J. \& KMENT P. 2018. The first record of the arachnophile stilt bug Metacanthus annulosus (Hemiptera: Heteroptera: Berytidae) in the Czech Republic, and its rediscovery in Slovakia. Klapalekiana 54: 123-130.

Wachmann E., Melber A. \& Deckert J. 2007. Wanzen. Band 3. Pentatomomorpha I. Aradidae, Lygaeidae, Piesmatidae, Berytidae, Pyrrhocoridae, Alydidae, Coreidae, Rhopalidae, Stenocephalidae. Tierwelt Deutschlands, 78, Goecke \& Evers, Keltern, 272 pp.

WAGNer E. 1941. Beitrag zur Heteropterenfauna Pommerns. Dohrniana 20: 33-78.

WRZESIŃSKA D., WAWRZYNIAK M. \& PIESIK D. 2013. Hemiptera infesting willow (Salix viminalis) plantations. Postępy w Ochronie Roślin 53 (1): 78-83. [In Polish with Enaglish Summary]

ZIARKIEWICZ T. 1957. Hemiptera-Heteroptera occuring on cruciferous plants in the Lublin District. Annales Universitatis Mariae Curie-Skłodowska, C, 12: 403-453. [In Polish with Russian Summary]

ZIARKIEWICZ T. 1958. Badania nad występowaniem pluskwiaków różnoskrzydłych (Hemiptera-Heteroptera) na uprawach ziemniaka na terenie Wandzina. Annales Universitatis Mariae Curie-Skłodowska, C, 13: 95-109.

ZIARKIEWICZ T. 1962. Investigations on color sensitivity in Hemiptera-Heteroptera, Neuroptera, Hymenoptera and Diptera. Annales Universitatis Mariae Curie-Skłodowska, C, 17: 77-108.

\section{STRESZCZENIE}

\section{[Smukleńcowate (Hemiptera: Heteroptera: Berytidae) Polski: lista gatunków, rozmieszczenie, bionomia]}

W Polsce pluskwiaki różnoskrzydłe $\mathrm{z}$ rodziny smukleńcowatych (Berytidae) reprezentowane są przez dziewięć gatunków sklasyfikowanych w czterech rodzajach: Neides tipularius, Berytinus clavipes, B. hirticornis, B. minor, B. crassipes, B. signoreti, Gampsocoris culicinus, G. punctipes i Metatropis rufescens. Niniejsze opracowanie, poza nowymi stanowiskami, podsumowuje wszystkie dane na temat występowania przedstawicieli Berytidae na terenie naszego kraju. Dodatkowo podano podstawowe informacje dotyczące biologii wymienionych gatunków (preferencje wilgotnościowe, termiczne, troficzne oraz ilość generacji w roku). Berytinus montivagus został wyłączony z krajowej fauny, jednak jego występowanie w Polsce (podobnie jak 5 innych gatunków notowanych w krajach ościennych) jest możliwe, zwłaszcza w dobie ocieplającego się klimatu. 